\title{
A Theoretical and Experimental Analysis of Macrodecoration of Defects in Monocrystalline Silicon
}

\author{
Milind S. Kulkarni,*,z Jeffrey Libbert,* Steven Keltner, \\ and Luciano Muléstagno
}

MEMC Electronic Materials, Incorporated, Saint Peters, Missouri 63376-0008, USA

\begin{abstract}
Microdefect distribution in a monocrystalline silicon wafer is identified by saturating the wafer with copper at a high temperature followed by copper precipitate growth through rapid cooling followed by surface polishing and subsequent microdefect-decorating etching. The decorated microdefect field consists of etch pits that are formed by the difference in the etching rates of the precipitate influenced region around microdefects, and the etching rate of the surrounding defect-free silicon. Interplay between liquid-phase mass-transport effects and surface kinetics plays a key role in the microdefect decoration. It is shown that the macrodecoration of microdefects is typically realized in the absence of significant effects of the liquid-phase diffusion. The developed phenomenological model leads to classification of etchants as either polishing or potentially decorating and to identification of conditions necessary for an efficient microdefect decoration. The competing effects of kinetics toward microdefect decoration and liquidphase transport toward surface polishing are quantified by theoretically derived solutions for the decorating efficiency and the polishing efficiency. Autoerosion of the microdefects by mildly polishing etchants is also quantified. Analytical expressions for the microdefect-decorating and microdefect-polishing conditions are presented. Finally, decorating and polishing etchants are experimentally identified from a group of etchants and the proposed theory is verified by experimental data.

(C) 2002 The Electrochemical Society. [DOI: 10.1149/1.1433473] All rights reserved.
\end{abstract}

Manuscript submitted May 11, 2001; revised manuscript received October 13, 2001. Available electronically January 17, 2002.

Defects in monocrystalline silicon.-The silicon crystal inherently contains many crystallographic imperfections. The most common type of imperfections in the crystals grown prior to the $1950 \mathrm{~s}$ were dislocations primarily formed by thermomechanical stresses in the vicinity of the melt/crystal interface. The major breakthrough by Dash $(1958,1959)$ allowed growth of dislocation-free crystals. ${ }^{1,2}$ However, elimination of thermomechanically induced dislocations introduced various types of crystallographic imperfections popularly known as microdefects. The microdefects are primarily of two origins: vacancy and self-interstitial. ${ }^{3}$ Vacancies are formed by a missing atom in the crystal lattice and a self-interstitial is a silicon atom that is displaced from its normal lattice position. Both vacancies and self-interstitials, which are collectively termed as point defects, exist as solutes in the bulk silicon solvent. The solubility of point defects exponentially increases with the temperature. ${ }^{3,4}$ As a segment of a growing crystal moves away from the melt/crystal interface, the temperature of the crystal segment decreases, allowing the gradual supersaturation of the point defects. Dislocation loops formed by thermomechanical stress provide sufficient surface area for absorption of the point defects. However, in the absence of such surface area, both self-interstitials and vacancies homogeneously agglomerate to form precipitates, which are known as microdefects. Abe and co-workers (1966) were first to report interstitial related defects, although the origin of these defects was not clearly known. ${ }^{5}$ These defects were later known as A-defects and B-defects, and were identified as interstitial-related dislocation loops and three-dimensional interstitial agglomerates, respectively. ${ }^{6-8}$ Vacancy agglomerates were first reported by Roksnoer and van den Boom (1981), which were later termed D-defects. ${ }^{9}$

History of microdefect decoration.-As the size of devices decreases in the current microelectronics industry, the demand on the quality of the silicon substrate becomes more stringent. The size and the spatial distribution of both vacancy and interstitial-type agglomerated microdefects play a critical role in device failure. Therefore, the quality of a silicon wafer is typically defined by the size and distribution of the microdefects. Device manufacturers have varying wafer-quality requirements and the challenge of producing different types of silicon wafers to meet different device makers' specifications is on the wafer manufacturer. The research, development, and

\footnotetext{
* Electrochemical Society Active Member

${ }^{\mathrm{z}}$ E-mail: mkulkarni@memc.com
}

subsequent production of a silicon wafer of the required quality involve identification and characterization of the microdefects.

Chemical etching is a popular method of microdefect characterization. Silicon etching, in essence, is a surface reaction that involves chemical dissolution of silicon into a liquid etchant. Most popular etchants used in microdefect decoration are mixtures of an oxidant, a chemical dissolving agent (generally, hydrofluoric acid) and inert diluents. Etching proceeds as described by the following global reactions ${ }^{4,10}$

$$
\begin{gathered}
\mathrm{Si}+\text { oxidant } \rightarrow \mathrm{SiO}_{2}+\text { gases }+ \text { by-products } \\
\mathrm{SiO}_{2}+6 \mathrm{HF} \rightarrow \mathrm{H}_{2} \mathrm{SiF}_{6}+2 \mathrm{H}_{2} \mathrm{O}
\end{gathered}
$$

Local stress levels at the microdefects are different from stress levels in the perfect silicon lattice. A class of etchants known as microdefect-decorating etchants, or simply, decorating etchants, shows sensitivity to this difference in the stress levels. In the presence of a decorating etchant, etching rates of the microdefects are different from etching rates of the perfect lattice. This differential etching creates either pits known as etch pits or hillocks at the microdefect sites, which then can be identified under a microscope. Oxidants and diluents used in an etching mixture influence its microdefect-decorating capacity. The popular oxidants used are $\mathrm{HNO}_{3}, \mathrm{CrO}_{3}, \mathrm{~K}_{2} \mathrm{Cr}_{2} \mathrm{O}_{7}$, and $\mathrm{Cu}\left(\mathrm{NO}_{3}\right)_{2}$. Most common diluents are $\mathrm{H}_{2} \mathrm{O}, \mathrm{CH}_{3} \mathrm{COOH}$, and other acids. Sirtl and Adler (1961), Secco d'Aragona (1972), Schimmel (1976), Jenkins (1977), and Yang (1984) were among many others who successfully experimented with various compositions of oxidants, HF, and diluents to characterize microdefects. ${ }^{11-15}$

Microdefect decoration is also accomplished by forced precipitation of selective metal solutes on the microdefects. Typically, metals precipitate as silicides. The technique involves saturation of the silicon wafer with a metal impurity at a high temperature followed by rapid cooling to allow supersaturation of the impurity and its subsequent precipitation on the microdefects. The most frequently used impurity to decorate microdefects in silicon wafers is copper. ${ }^{16}$ The copper decorated microdefects can be characterized by optical or infrared microscopy (Dash, 1956), transmission X-ray topography (XRT, de Kock, 1973) or by the Lang XRT method (de Kock, 1970). ${ }^{16-18}$ Considerable progress has been made in characterizing copper precipitates (recognized as $\mathrm{Cu}_{3} \mathrm{Si}$ ) since the 1970 s and the method of copper precipitation for microdefect decoration has been very well established. ${ }^{19,20}$ 
In the past, techniques of copper precipitation and decorating etching for microdefect characterization were not applied in conjunction with each other. The density of a copper precipitate is much lower than that of silicon. Therefore, growth of a copper precipitate generates compressive stresses in the surrounding area. These stresses are relieved by ejection of silicon lattice atoms as interstitials. The ejected interstitials form new dislocations around the copper precipitate. ${ }^{20}$ Subsequent copper precipitation can take place on these dislocations. This precipitate-influenced region, containing many copper precipitates and dislocations formed around a single microdefect, is identified as a copper precipitate colony or copper colony. Different metals can form different precipitate colonies. A copper precipitate colony is much larger in size than the original microdefect. ${ }^{20}$ After copper precipitation, a microdefect site is identified by a much enlarged region occupied by the associated copper precipitate colony. Hence, a more efficient characterization of microdefects is accomplished by copper precipitation followed by decorating etching. ${ }^{21}$

Limitations of the current knowledge.-Chemical etching is a multiphase reaction that involves diffusion of reagents in the liquid phase and subsequent reaction on the wafer surface. Hence, the etching rate of any site on the wafer surface is described by both liquidphase diffusion of reagents and surface kinetics. Both kinetics and liquid-phase diffusion (mass-transport) determine local etching rates and, thereby, the quality of microdefect decoration. The masstransport effects are present in many different etching systems. Schwartz and Robbins (1961, 1976), Robbins and Schwartz (1959, 1960), Bogenschütz et al. (1967), and Klein and D'Stefan (1962) are among many others to report the presence of mass-transport effects in various etching systems. ${ }^{22-27}$ Generally, the presence of mass-transport influence was studied by observing the etching rate variation with the mixing intensity and the temperature of the liquid phase. The dependence of the saturation current density of an n-type silicon electrode on the intensity of mixing was reported by Turner $(1961) .{ }^{28}$ In their study to understand stain reduction, Gaffney and Chiou (1998) reported mass-transport effects in acid-based etching. ${ }^{29}$ Bauer et al. (1998) reported the importance of fluid dynamics in etching but offered no quantitative or phenomenological explanation. ${ }^{30}$ John and McDonald (1993) observed the dependence of spray etching performance on the design of the etching equipment, which seems to indicate the influence of mass transport. ${ }^{31}$

In a study on the dissolution of thermal oxide of silicon by HF, Monk et al. (1994) revealed the shift from kinetics to diffusion in the rate controlling step. ${ }^{32,33}$ Monk et al. (1993) also studied the kinetics of dissolution of thermal oxide of silicon by HF. ${ }^{34}$ Although these studies did not address the in situ oxidation of silicon by an oxidant and simultaneous dissolution by HF, the potential significance of mass-transport effects in such systems was revealed. The presence of diffusion effects in dissolution of thermal oxide by HF solutions of various compositions were acknowledged by Monk and Soane (1993). ${ }^{35}$

In current literature, there is an abundance of information on both copper precipitation and decorating etching. However, current understanding of microdefect decoration by etching is based on the difference between the etching rates of the microdefects and of perfect silicon. Generally, this difference is attributed to the difference in the kinetics between the two regions. However, kinetics describes only surface reactions. Kulkarni and Erk (2000) proposed a generic theory of etching that incorporates both mass-transfer and kinetic effects. ${ }^{36}$ In essence, they showed that the presence of liquid-phase diffusion effects typically reduces the intensity of surface irregularities such as roughness and resists formation of surface irregularities because of a polishing effect. Since the etch pits formed by a decorating etchant are also surface irregularities, it can be argued that the presence of significant mass-transport effects can affect the quality of microdefect decoration. The relative magnitude of the diffusion time scale compared to that of the kinetic time scale is the key parameter that describes the quality of microdefect decoration. So far, no concise reported model in the literature can relate the quality of microdefect decoration to both diffusion and kinetic effects. Therefore, there is a need to study and quantify the quality of microdefect decoration by incorporating these effects. It is also necessary to identify parameters that determine the nature of an etchant as either polishing or decorating.

\section{Quantification of Process Time Scales}

Chemical etching of silicon wafers is a three-phase system that involves liquid-phase diffusion of reactants from the bulk liquid to the wafer surface, surface reactions that generate products in both the gas phase and the liquid phase, and subsequent transport of products into the bulk liquid. ${ }^{36}$ Effects of gaseous bubbles on the surface morphology can be incorporated by the assumption of a pseudoliquid phase with an effective diffusivity. According to the classical film-transport theory, the diffusion of reagents occurs through a stagnant film (concentration boundary layer) and thus, the mass-transport rate per unit area of the wafer is defined as ${ }^{36-39}$

$$
r_{\mathrm{m}, \mathrm{i}}=\frac{D_{\mathrm{eff}, \mathrm{i}}}{\delta_{\mathrm{eff}, \mathrm{i}}}\left(C_{\mathrm{b}, \mathrm{i}}-C_{\mathrm{f}, \mathrm{i}}\right)=k_{\mathrm{m}, \mathrm{eff}, \mathrm{i}}\left(C_{\mathrm{b}, \mathrm{i}}-C_{\mathrm{f}, \mathrm{i}}\right)
$$

where $r_{\mathrm{m}}\left(\mathrm{mol} / \mathrm{m}^{2} \mathrm{~s}\right)$ is the rate of transport of a given reagent per unit surface area, $D\left(\mathrm{~m}^{2} / \mathrm{s}\right)$ is the diffusivity of a given reagent in the liquid film, $\delta(\mathrm{m})$ is the liquid film thickness, $C\left(\mathrm{~mol} / \mathrm{m}^{3}\right)$ is the concentration of a given reagent and $k_{\mathrm{m}}(\mathrm{m} / \mathrm{s})$ is the mass-transport coefficient for a given reagent. Subscript $m$ denotes mass transport, $\mathrm{b}$ refers to the bulk liquid conditions, $\mathrm{f}$ refers to the wafer surface conditions, and i identifies the reagent. Phenomenological parameters are identified by subscript eff. Depending on the complexity of hydrodynamics an effective thickness of the liquid film can be defined. Throughout this paper, the term transport-film thickness denotes the effective thickness of this film. The rate of consumption of a given reagent by the surface reaction per unit area is given by

$$
r_{\mathrm{r}, \mathrm{i}}=f_{\mathrm{r}}\left(T, C_{\mathrm{f}, \mathrm{i}}, C_{\mathrm{f}, \mathrm{j}}, C_{\mathrm{f}, \mathrm{k}} \ldots\right)
$$

where $r_{\mathrm{r}}\left(\mathrm{mol} / \mathrm{m}^{2} \mathrm{~s}\right)$ is the rate of consumption (not rate of formation) of a given reagent per unit area, $T(\mathrm{~K})$ is the absolute temperature, and $f_{\mathrm{r}}$ is a function describing the surface kinetics. Subscript $\mathrm{r}$ refers to reaction and subscripts $\mathrm{j}, \mathrm{k}$... denote relevant species on the wafer surface that take part in the etching reaction.

It can be shown that at pseudosteady state the overall rate of etching is given by

$$
\begin{aligned}
r_{\mathrm{i}} & =r_{\mathrm{m}, \mathrm{i}}=r_{\mathrm{r}, \mathrm{i}}=\frac{D F_{\mathrm{o}, \mathrm{i}}}{R_{\mathrm{o}, \mathrm{eff}, \mathrm{i}}}=\frac{D F_{\mathrm{m}, \mathrm{i}}}{R_{\mathrm{m}, \mathrm{eff}, \mathrm{i}}}=\frac{D F_{\mathrm{r}, \mathrm{i}}}{R_{\mathrm{r}, \mathrm{eff}, \mathrm{i}}} \\
& \rightarrow R_{\mathrm{o}, \mathrm{eff}, \mathrm{i}}=R_{\mathrm{m}, \mathrm{eff}, \mathrm{i}}+R_{\mathrm{r}, \mathrm{eff}, \mathrm{i}}
\end{aligned}
$$

where

$$
\begin{gathered}
D F_{\mathrm{o}, \mathrm{i}}=C_{\mathrm{b}, \mathrm{i}} \\
D F_{\mathrm{m}, \mathrm{i}}=C_{\mathrm{b}, \mathrm{i}}-C_{\mathrm{f}, \mathrm{i}} \\
D F_{\mathrm{r}, \mathrm{i}}=C_{\mathrm{f}, \mathrm{i}} \\
R_{\mathrm{m}, \mathrm{eff}, \mathrm{i}}=\frac{\delta_{\mathrm{eff}, \mathrm{i}}}{D_{\mathrm{eff}, \mathrm{i}}}=\frac{1}{k_{\mathrm{m}, \mathrm{eff}, \mathrm{i}}} \\
R_{\mathrm{r}, \mathrm{eff}, \mathrm{i}}=\frac{1}{\left[\frac{f_{\mathrm{r}}\left(T, C_{\mathrm{f}, \mathrm{i}}, C_{\mathrm{f}, \mathrm{j}}, C_{\mathrm{f}, \mathrm{k}} \cdots\right)}{C_{\mathrm{f}, \mathrm{i}}}\right]}
\end{gathered}
$$

Here $r_{\mathrm{i}}\left(\mathrm{mol} / \mathrm{m}^{2} \mathrm{~s}\right)$, the overall rate of consumption of a reactant $\mathrm{i}$, is given as the ratio of a driving force, $D F\left(\mathrm{~mol} / \mathrm{m}^{3}\right)$, to a resistance, $R$ $(\mathrm{s} / \mathrm{m})$. The driving force and the resistance can be based on only 
mass transport, only kinetics, or both. Subscript o indicates overall effect of kinetics as well as mass transport. Kulkarni and Erk (2000) defined a kinetic resistance based on the uniform interfacial concentration predicted by the effective mass-transport resistance and the rate of transport. Thus, true kinetic parameters could be used to define the kinetic resistance. However, in this study, we define an effective kinetic resistance for better accuracy. Throughout this paper, the term kinetics refers to the effective kinetics.

The rate of decrease in the wafer thickness and the rate of consumption of a reactant are related by stoichiometry of the etching reaction and the wafer density

$$
r l=\frac{n_{\mathrm{i}}}{\rho_{\text {molar }}} r_{\mathrm{i}}=\xi_{\mathrm{i}} r_{\mathrm{i}}
$$

where $r l(\mathrm{~m} / \mathrm{s})$ is the rate of decrease (not rate of increase) in the wafer thickness or the linear removal rate, $n_{\mathrm{i}}$ is the number of moles of silicon (or reacting solid) reacted per mole of reactant $i$ consumed, $\rho_{\text {molar }}\left(\mathrm{mol} / \mathrm{m}^{3}\right)$ is the molar density of silicon (or reacting solid), and $\xi\left(\mathrm{m}^{3} / \mathrm{mol}\right)$ is the conversion factor. Using Eq. 5 and 6 , the resistances based on the rate of decrease in the wafer thickness are given by

$$
r l=\frac{D F_{\mathrm{o}, \mathrm{i}}}{\beta_{\mathrm{o}, \mathrm{eff}, \mathrm{i}}}=\frac{D F_{\mathrm{m}, \mathrm{i}}}{\beta_{\mathrm{m}, \mathrm{eff}, \mathrm{i}}}=\frac{D F_{\mathrm{r}, \mathrm{i}}}{\beta_{\mathrm{r}, \mathrm{eff}, \mathrm{i}}} \rightarrow \beta_{\mathrm{o}, \mathrm{eff}, \mathrm{i}}=\beta_{\mathrm{m}, \mathrm{eff}, \mathrm{i}}+\beta_{\mathrm{r}, \mathrm{eff}, \mathrm{i}}
$$

where

$$
\beta_{\mathrm{o}, \mathrm{eff}, \mathrm{i}}=\frac{R_{\mathrm{o}, \mathrm{eff}, \mathrm{i}}}{\zeta_{\mathrm{i}}} \quad \beta_{\mathrm{m}, \mathrm{eff}, \mathrm{i}}=\frac{R_{\mathrm{m}, \mathrm{eff}, \mathrm{i}}}{\zeta_{\mathrm{i}}}
$$

and

$$
\beta_{\mathrm{r}, \mathrm{eff}, \mathrm{i}}=\frac{R_{\mathrm{m}, \mathrm{eff}, \mathrm{i}}}{\xi_{\mathrm{i}}}
$$

$\beta\left(\mathrm{mol} \mathrm{s} / \mathrm{m}^{4}\right)$ is the resistance based on the rate of decrease in the wafer thickness.

Resistance is a quantitative measure of the time scale of a process. Thus, the effective mass-transport resistance reflects the masstransport time scale, the effective kinetic resistance reflects the surface kinetic time scale and the effective overall resistance reflects the time scale of the overall process. It is clear from Eq. 5 and 7 that the effective overall resistance of the process is a linear combination of the effective kinetic resistance and the effective mass-transport resistance. Since etching takes place through a mass-transport step and a kinetic step, the rate-controlling step is determined by the magnitude of the ratio of the effective mass-transport resistance to the effective kinetic resistance.

\section{Microdefect Decoration}

Procedure.-In this study, advantages of copper precipitation as well as decorating etching are combined by a hybrid decorating technique. The following steps are followed sequentially.

1. Saturation of the silicon wafer with copper at a temperature above $800^{\circ} \mathrm{C}$.

2. Rapid cooling of the silicon wafer to allow heterogeneous nucleation of copper on the microdefects and subsequent growth of copper precipitate colonies.

3. Chemical surface polishing to clean the wafer surface.

4. Decorating etching to decorate the copper precipitate colonies.

\section{Quantification of Microdefect Decoration}

Microdefect decoration takes place because the etching rates (more precisely, dissolution rates) of copper precipitate colonies are greater than the etching rates of surrounding perfect silicon. In this work, only copper precipitate enhanced microdefect decoration is treated. Thus, each copper precipitate colony represents a microde-

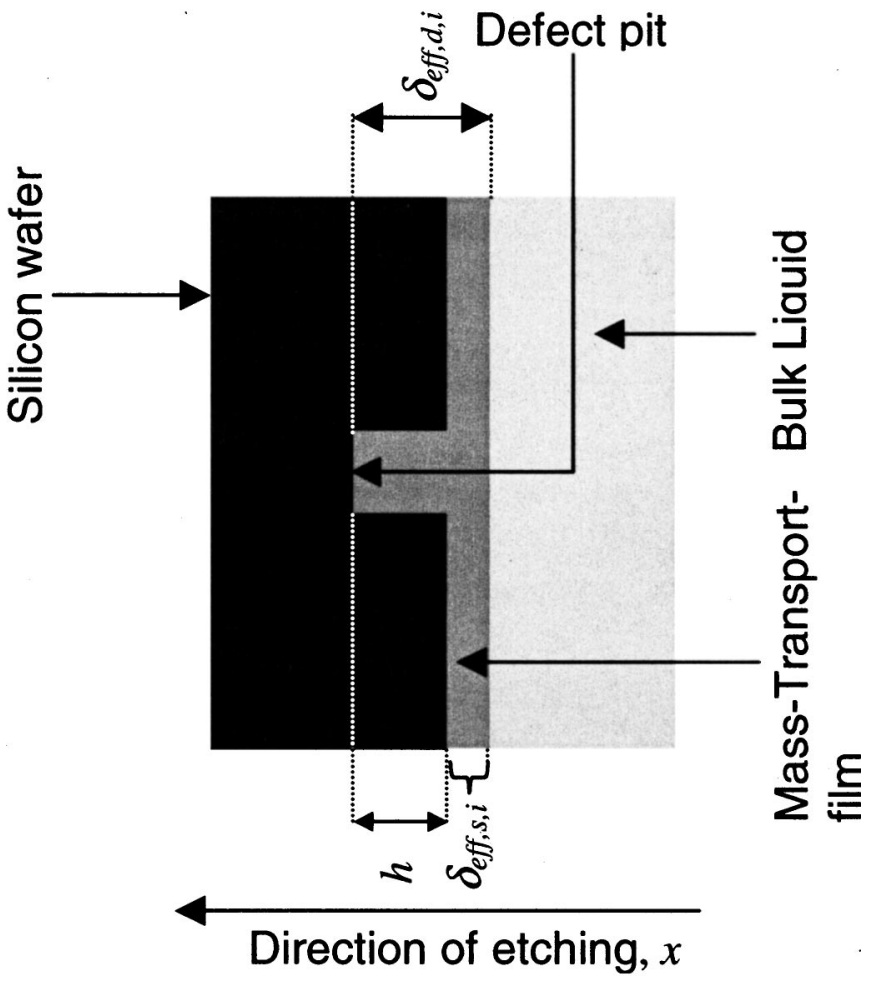

Figure 1. A phenomenological representation of microdefect decoration by etching.

fect. Figure 1 shows a part of a silicon wafer with a microdefect related etch pit of depth $h(\mathrm{~m})$ covered by a stagnant liquid-film of thickness $\delta_{\text {eff,s,i }}(\mathrm{m})$ on the surface and $\delta_{\text {eff,d,i }}(\mathrm{m})$ on the pit. Although etching takes place in all directions, for generic simplicity, it is assumed that effective etching takes place predominantly in the $x$ direction. The rate of increase in the pit depth is given by the difference between the linear removal rates of the silicon surface and the copper precipitate colony

$$
\frac{d h}{d t}=r l_{\mathrm{d}}-r l_{\mathrm{s}}
$$

where

$$
\begin{aligned}
& r l_{\mathrm{d}}=\xi_{\mathrm{d}, \mathrm{i}} r_{\mathrm{d}, \mathrm{i}} \\
& r l_{\mathrm{s}}=\xi_{\mathrm{s}, \mathrm{i}} r_{\mathrm{s}, \mathrm{i}}
\end{aligned}
$$

where subscript d denotes the copper precipitate colony and the etch pit formed by it, and s denotes the perfect silicon surface. Subscript d also denotes the pit after complete dissolution of the copper precipitate colony. The maximum rate at which the pit depth can increase is given by

$$
\left[\frac{d h}{d t}\right]_{\max }=r l_{\mathrm{d}}
$$

The decorating efficiency can be defined as the ratio of the rate of increase in the pit depth to the maximum possible rate of increase in the pit depth 


$$
\begin{aligned}
& I=\frac{\left(\frac{d h}{d t}\right)}{\left[\frac{d h}{d t}\right]_{\max }} \\
& =1-\frac{\beta_{\mathrm{r}, \mathrm{eff}, \mathrm{d}, \mathrm{i}}}{\beta_{\mathrm{r}, \mathrm{eff}, \mathrm{s}, \mathrm{i}}}\left[\frac{1+\left(\frac{\beta_{\mathrm{m}, \mathrm{eff}, \mathrm{d}, \mathrm{i}}}{\beta_{\mathrm{r}, \mathrm{eff}, \mathrm{d}, \mathrm{i}}}\right)}{1+\left(\frac{\beta_{\mathrm{m}, \mathrm{eff}, \mathrm{s}, \mathrm{i}}}{\beta_{\mathrm{r}, \mathrm{eff}, \mathrm{s}, \mathrm{i}}}\right)}\right] \\
& =1-\frac{\xi_{\mathrm{s}, \mathrm{i}}}{\xi_{\mathrm{d}, \mathrm{i}}} \frac{R_{\mathrm{r}, \mathrm{eff}, \mathrm{d}, \mathrm{i}}}{R_{\mathrm{r}, \mathrm{eff}, \mathrm{s}, \mathrm{i}}}\left[\frac{1+\left(\frac{R_{\mathrm{m}, \mathrm{eff}, \mathrm{d}, \mathrm{i}}}{R_{\mathrm{r}, \mathrm{eff}, \mathrm{d}, \mathrm{i}}}\right)}{1+\left(\frac{R_{\mathrm{m}, \mathrm{eff}, \mathrm{s}, \mathrm{i}}}{R_{\mathrm{r}, \mathrm{eff}, \mathrm{s}, \mathrm{i}}}\right)}\right]
\end{aligned}
$$

The average decorating efficiency is the integral average of the decorating efficiency during the pit growth

$$
I_{\mathrm{avg}}=\frac{\int_{\mathrm{h}_{1}}^{\mathrm{h}_{2}}\left\{\left(\frac{d h}{d t}\right) /\left[\frac{d h}{d t}\right]_{\max }\right\} d h}{h_{2}-h_{1}}
$$

where subscripts 1 and 2 indicate the pit depth at the onset and end of decoration.

Equation 12 clearly demonstrates the significance of the liquidphase diffusion on microdefect decoration. It is evident that the microdefect decoration takes place only when the decorating efficiency is positive and that its quality is strongly influenced by the effective mass-transport resistance.

During decorating etching, the decorating efficiency remains positive and finite at a pit in the presence of a copper precipitate colony. After complete dissolution of the given copper precipitate colony at the pit, no further increase in the pit depth is possible, because kinetic resistance at the pit and that at the surface become almost identical. However, the geometry of the pit is preserved throughout the decorating etching process as the etching rate of the pit remains practically equal to the etching rate of the surface.

\section{Quantification of Surface Polishing}

Surface polishing by an etchant takes place when the etching rates of the pits or valleys on the wafer surface are lower than the etching rates of planar areas. With reference to Fig. 1, the polishing rate is defined as

$$
-\frac{d h}{d t}=r l_{\mathrm{s}}-r l_{\mathrm{d}}
$$

The maximum polishing rate is achieved when the linear removal rate at the pit, $r l_{\mathrm{d}}$, is equal to zero

$$
\left[-\frac{d h}{d t}\right]_{\max }=r l_{\mathrm{s}}
$$

The polishing efficiency of any etchant is defined as the rate of decrease in the pit depth to the maximum possible rate of decrease. It must be noted that the polishing efficiency at an etch pit in the presence of an associated copper precipitate colony is lower than the polishing efficiency in its absence. In general, the polishing efficiency is given by the following equation

$$
\begin{aligned}
\eta & =\frac{-\left(\frac{d h}{d t}\right)}{\left[-\frac{d h}{d t}\right]_{\max }} \\
& =1-\frac{\beta_{\mathrm{r}, \mathrm{eff}, \mathrm{s}, \mathrm{i}}}{\beta_{\mathrm{r}, \mathrm{eff}, \mathrm{d}, \mathrm{i}}}\left[\frac{1+\left(\frac{\beta_{\mathrm{m}, \mathrm{eff}, \mathrm{s}, \mathrm{i}}}{\beta_{\mathrm{r}, \mathrm{eff}, \mathrm{s}, \mathrm{i}}}\right)}{\left.1+\left(\frac{\beta_{\mathrm{m}, \mathrm{eff}, \mathrm{d}, \mathrm{i}}}{\beta_{\mathrm{r}, \mathrm{eff}, \mathrm{d}, \mathrm{i}}}\right)\right]}\right. \\
& =1-\frac{\xi_{\mathrm{d}, \mathrm{i}}}{\xi_{\mathrm{s}, \mathrm{i}}} \frac{R_{\mathrm{r}, \mathrm{eff}, \mathrm{s}, \mathrm{i}}}{R_{\mathrm{r}, \mathrm{eff}, \mathrm{d}, \mathrm{i}}}\left[\frac{1+\left(\frac{R_{\mathrm{m}, \mathrm{eff}, \mathrm{s} \mathrm{i}}}{R_{\mathrm{r}, \mathrm{eff}, \mathrm{s}, \mathrm{i}}}\right)}{1+\left(\frac{R_{\mathrm{m}, \mathrm{eff}, \mathrm{d}, \mathrm{i}}}{R_{\mathrm{r}, \mathrm{eff}, \mathrm{d}, \mathrm{i}}}\right)}\right]
\end{aligned}
$$

The average polishing efficiency of an etchant is the integral average of the polishing efficiency from $h_{1}$ to $h_{2}$

$$
\eta_{\text {avg }}=\frac{\int_{\mathrm{h}_{1}}^{\mathrm{h}_{2}}\left\{\left(-\frac{d h}{d t}\right) /\left[-\frac{d h}{d t}\right]_{\max }\right\} d h}{h_{2}-h_{1}}
$$

It is evident that the polishing efficiency in the presence of the copper precipitate colonies must be negative for microdefect decoration to take place. However, in the presence of the liquid-phase diffusion effects, the polishing effect may dominate. Microdefect decoration cannot proceed when the polishing efficiency in the presence of a copper precipitate colony is positive. The polishing efficiency and the decorating efficiency have opposite signs except when both are equal to zero.

\section{Microdefect Polishing and Normal Polishing}

It is important to recognize the distinction between microdefect polishing and normal polishing. Polishing at the microdefect site or microdefect polishing is resisted by the difference between the intrinsic etching kinetics of the native microdefect or the copper precipitate colony and that of perfect silicon (i.e., $\beta_{\mathrm{r}, \text { eff,d,i }}$ and $\beta_{\mathrm{r}, \mathrm{eff}, \mathrm{s}, \mathrm{i}}$ are described by different intrinsic kinetics). Normal polishing takes place over a rough silicon surface in the absence of microdefects or copper precipitates and is described by the same intrinsic kinetics. Thus, for the normal polishing, the intrinsic kinetics of the etched area remains uniform (i.e., $\beta_{\mathrm{r}, \text { eff,d,i }}$ and $\beta_{\mathrm{r}, \mathrm{eff}, \mathrm{s}, \mathrm{i}}$ are described by the same intrinsic kinetics). An etchant can exhibit a negative polishing efficiency (positive decorating efficiency) in the presence of a copper precipitate colony and a positive polishing efficiency on the rough silicon surface. Under such conditions, small and shallow etch pits are formed in the presence of the copper precipitates. However, these small pits are erased after the copper precipitates are completely etched. Thus, an etch pit can make a transition from decorating conditions to polishing conditions. Typically, this transition takes place upon complete dissolution of the copper precipitate colony.

\section{Analysis of Microdefect Decoration and Surface Polishing}

Equations 12 and 16 describe the generic effect of the mass transport and the surface kinetics on any etching process. Microdefects are decorated when the decorating efficiencies at the microdefect sites are positive. Surface irregularities including the microdefect related etch pits are eventually erased if the normal polishing takes place. Equations 12 and 16 describe microdefect decoration and surface polishing under all conditions. However, insight into the role of transport on microdefect decoration is better achieved by analyzing two limiting cases. For precipitate aided decorating etching, typically $\beta_{\mathrm{r}, \mathrm{eff}, \mathrm{d}, \mathrm{i}} / \beta_{\mathrm{r}, \mathrm{eff}, \mathrm{s}, \mathrm{i}}$ is less than 1 . The following discussion focuses on this case. However, the general argument can be extended to all conditions. 

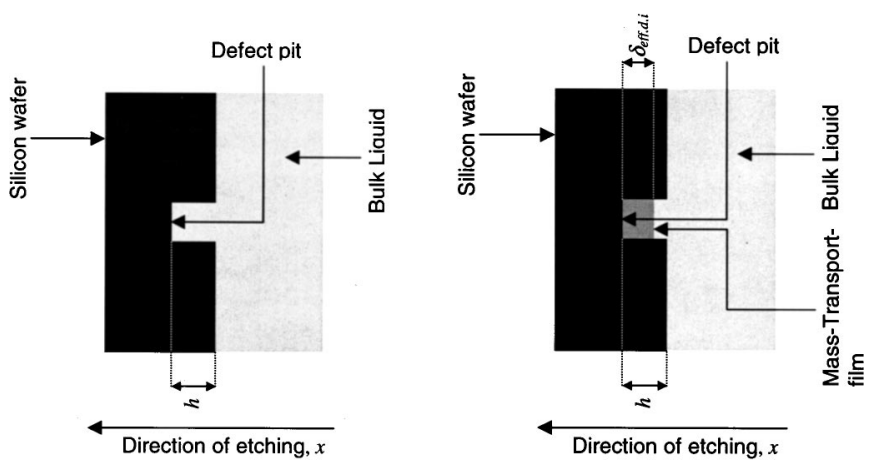

(a)

(b)
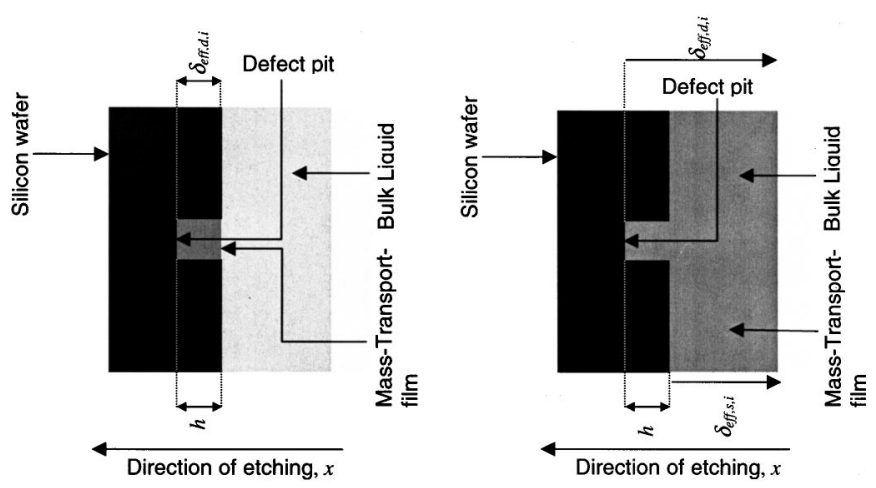

(c)

(d)

Figure 2. A phenomenological representation of microdefect decoration (a) in the absence of the transport film, (b) in the presence of a stagnant cell in the etch pit, (c) at $h=\delta_{\text {eff,d,i }}$ and $\delta_{\text {eff, }, \mathrm{i}}=0$, and (d) for infinite masstransport film thickness.

Limiting case 1: Effect of the transport-film thickness.-The mass-transport time scale and the effective mass-transport resistance each increase with increasing thickness of the transport film. The effective thickness of this film is a function of the etchant composition and the hydrodynamic conditions in the etching reactor. The transport-film thickness decreases with increasing mixing intensity in a reactor. ${ }^{38,39}$ Thus, the film thickness decreases with increasing mixing intensity in the etching reactor. Consider a hypothetical scenario shown in Fig. 2. The film thickness is negligible in Fig. 2a. The etching is kinetically controlled, the decorating efficiency at the microdefect site (etch pit) is at its highest (maximum) value, and the polishing efficiency at the site is at its lowest (minimum) value

$$
\begin{array}{r}
\lim _{\beta_{\mathrm{m}, \mathrm{eff}, \mathrm{s}, \mathrm{i}} \rightarrow 0, \beta_{\mathrm{m}, \mathrm{eff}, \mathrm{d}, \mathrm{i}} \rightarrow 0} I=1-\frac{\xi_{\mathrm{s}, \mathrm{i}}}{\xi_{\mathrm{d}, \mathrm{i}}} \frac{R_{\mathrm{r}, \mathrm{eff}, \mathrm{d}, \mathrm{i}}}{R_{\mathrm{r}, \mathrm{eff}, \mathrm{s}, \mathrm{i}}}=1-\frac{\beta_{\mathrm{r}, \mathrm{eff}, \mathrm{d}, \mathrm{i}}}{\beta_{\mathrm{r}, \mathrm{eff}, \mathrm{s}, \mathrm{i}}} \\
\lim _{\beta_{\mathrm{m}, \mathrm{eff}, \mathrm{s}, \mathrm{i}} \rightarrow 0, \beta_{\mathrm{m}, \mathrm{eff}, \mathrm{i}, \rightarrow} \rightarrow 0} \eta=1-\frac{\xi_{\mathrm{d}, \mathrm{i}}}{\xi_{\mathrm{s}, \mathrm{i}}} \frac{R_{\mathrm{r}, \mathrm{eff}, \mathrm{s}, \mathrm{i}}}{R_{\mathrm{r}, \mathrm{eff}, \mathrm{d}, \mathrm{i}}}=1-\frac{\beta_{\mathrm{r}, \mathrm{eff}, \mathrm{s}, \mathrm{i}}}{\beta_{\mathrm{r}, \mathrm{eff}, \mathrm{d}, \mathrm{i}}}
\end{array}
$$

As the hydrodynamic conditions in the reactor change, stagnant cells in the shallow etch pits can develop, as shown in Fig. 2b. These cells can be represented as an effective film that covers part of the pit. Under this condition, the decorating and polishing efficiency at the etch pit at the microdefect site are given by

$$
\begin{aligned}
I & =1-\frac{\xi_{\mathrm{s}, \mathrm{i}}}{\xi_{\mathrm{d}, \mathrm{i}}} \frac{R_{\mathrm{r}, \mathrm{eff}, \mathrm{d}, \mathrm{i}}}{R_{\mathrm{r}, \mathrm{eff}, \mathrm{s}, \mathrm{i}}}\left[1+\frac{R_{\mathrm{m}, \mathrm{eff}, \mathrm{d}, \mathrm{i}}}{R_{\mathrm{r}, \mathrm{eff}, \mathrm{d}, \mathrm{i}}}\right] \\
& =1-\frac{\beta_{\mathrm{r}, \mathrm{eff}, \mathrm{d}, \mathrm{i}}}{\beta_{\mathrm{r}, \mathrm{eff}, \mathrm{s}, \mathrm{i}}}\left[1+\frac{\beta_{\mathrm{m}, \mathrm{eff}, \mathrm{d}, \mathrm{i}}}{\beta_{\mathrm{r}, \mathrm{eff}, \mathrm{d}, \mathrm{i}}}\right] \\
\eta & =1-\frac{\xi_{\mathrm{d}, \mathrm{i}}}{\xi_{\mathrm{s}, \mathrm{i}}} \frac{R_{\mathrm{r}, \mathrm{eff}, \mathrm{s}, \mathrm{i}}}{R_{\mathrm{r}, \mathrm{eff}, \mathrm{d}, \mathrm{i}}}\left[\frac{1}{\left(1+\frac{R_{\mathrm{m}, \mathrm{eff}, \mathrm{d}, \mathrm{i}}}{R_{\mathrm{r}, \mathrm{eff}, \mathrm{d}, \mathrm{i}}}\right)}\right] \\
& =1-\frac{1}{\beta_{\mathrm{r}, \mathrm{efff}, \mathrm{d}, \mathrm{i}}}\left[\frac{1}{\left(1+\frac{\beta_{\mathrm{m}, \mathrm{eff}, \mathrm{d}, \mathrm{i}}}{\beta_{\mathrm{r}, \mathrm{eff}, \mathrm{d}, \mathrm{i}}}\right)}\right]
\end{aligned}
$$

Equation 20 indicates that the decorating efficiency decreases as the transport effects in the shallow pit increase. As the size of these stagnant cells gradually increases, the decorating efficiency decreases and becomes zero when the condition

$$
\beta_{\mathrm{r}, \mathrm{eff}, \mathrm{d}, \mathrm{i}}+\beta_{\mathrm{m}, \mathrm{eff}, \mathrm{d}, \mathrm{i}}-\beta_{\mathrm{r}, \mathrm{eff}, \mathrm{s}, \mathrm{i}}=0 \text { and } \beta_{\mathrm{m}, \mathrm{eff}, \mathrm{s}, \mathrm{i}}=0
$$

is met. Equation 22 indicates the crossover limit. This happens when the sum of the kinetic and transport resistances at the pit equals the sum of the kinetic and transport resistances on the perfect surface. Beyond this limit, in the absence of the effective mass-transport resistance on the surface (i.e., for $\beta_{\mathrm{m}, \text { eff }, \mathrm{s}, \mathrm{i}}=0$ ), increase in the effective mass-transport resistance at the shallow pit changes the etching condition from decorating to polishing.

A shift in the etchant behavior occurs when the surface masstransport resistance becomes finite (nonzero). The threshold of this shift is marked by the equality of the effective mass-transport film thickness on the pit and the pit depth $\left(\delta_{\text {eff, }, \mathrm{i}}=h\right)$ when the surface film thickness is still zero $\left(\delta_{\text {eff,s,i }}=0\right)$. The critical values of the decorating efficiency and the polishing efficiency at this shift (Fig. 2c) are still given by Eq. 20 and 21, respectively. When the stagnant transport film covers both the wafer surface and the pit as shown in Fig. 1, both the decorating efficiency and the polishing efficiency at the microdefect site are given by Eq. 12 and 16, respectively. As the film thickness approaches infinity (Fig. 2d), the decorating efficiency approaches an asymptotic value given by Eq. 23. The polishing efficiency approaches an asymptotic value given by Eq. 24 when the film thickness approaches infinity

$$
\begin{gathered}
\lim _{\delta_{\mathrm{eff}, \mathrm{s}, \mathrm{i}} \rightarrow \infty} I=1-\frac{\xi_{\mathrm{s}, \mathrm{i}}}{\xi_{\mathrm{d}, \mathrm{i}}} \\
\lim _{\delta_{\mathrm{eff}, \mathrm{s}, \mathrm{i}} \rightarrow \infty} \eta=1-\frac{\xi_{\mathrm{d}, \mathrm{i}}}{\xi_{\mathrm{s}, \mathrm{i}}}
\end{gathered}
$$

The behavior of an etchant as a function of mass-transport and kinetic effects at the microdefect site can be illustrated on a graph of

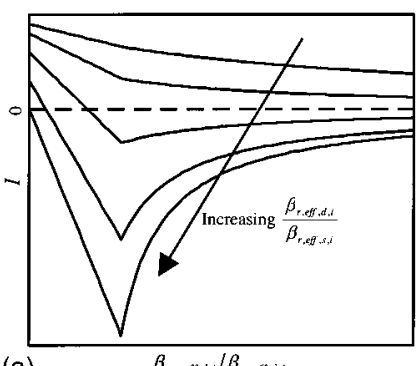

(a)

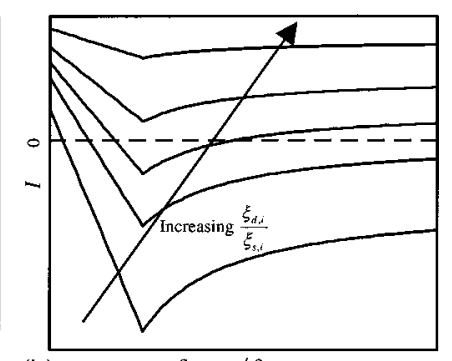

(b) $\beta_{m, e f f, d, i} / \beta_{r, e f i, d, i}$
Figure 3. Effect of the mass-transport film thickness on the decorating efficiency at a microdefect-site for a given pit depth and (a) $\xi_{\mathrm{d}, \mathrm{i}} / \xi_{\mathrm{s}, \mathrm{i}}$, (b) $R_{\mathrm{r}, \mathrm{eff}, \mathrm{d}, \mathrm{i}} / R_{\mathrm{r}, \mathrm{eff}, \mathrm{s}, \mathrm{i}}$. 

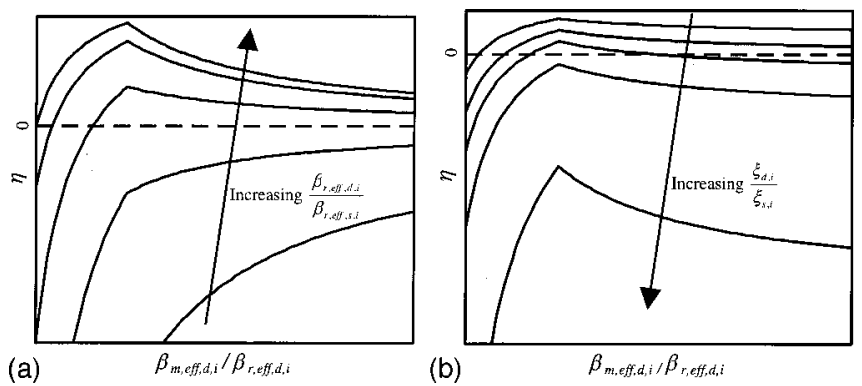

Figure 4. Effect of the mass-transport film thickness on the polishing efficiency at a microdefect site for a given pit depth and (a) $\xi_{\mathrm{d}, \mathrm{i}} / \xi_{\mathrm{s}, \mathrm{i}}$, (b) $R_{\mathrm{r}, \mathrm{eff}, \mathrm{d}, \mathrm{i}} / R_{\mathrm{r}, \mathrm{eff}, \mathrm{s}, \mathrm{i}}$.

decorating efficiency $(I) v s$. the ratio of mass-transport resistance to the kinetic resistance $\left(\beta_{\mathrm{m}, \mathrm{eff}, \mathrm{d}, \mathrm{i}} / \beta_{\mathrm{r}, \text { eff, d,i }}\right)$. Figure $3 \mathrm{a}$ shows a family of curves on this graph for $\xi_{\mathrm{d}, \mathrm{i}} / \xi_{\mathrm{s}, \mathrm{i}}=1$. Each curve on this graph is an isokinetic line that represents a fixed ratio of the time scale of the kinetics at the etch pit at the microdefect site to the time scale of the surface kinetics, $\beta_{\mathrm{r}, \mathrm{eff}, \mathrm{d}, \mathrm{i}} / \beta_{\mathrm{r}, \mathrm{eff}, \mathrm{s}, \mathrm{i}}$. It is important to note that an isokinetic line does not necessarily capture the variation of the decorating efficiency of a particular etchant. For a given etchant, the locus of the decorating efficiency as a function of $\beta_{\mathrm{m}, \mathrm{eff}, \mathrm{d}, \mathrm{i}} / \beta_{\mathrm{r}, \mathrm{eff}, \mathrm{d}, \mathrm{i}}$ does not necessarily represent a fixed $\beta_{\mathrm{r}, \mathrm{eff}, \mathrm{d,i}} / \beta_{\mathrm{r}, \mathrm{eff}, \mathrm{s}, \mathrm{i}}$, and hence, does not necessarily represent an isokinetic line. However, a given point on that locus lies on some isokinetic line. Thus, all etchants can be mapped on this graph. The kinetic tendency toward microdefect decoration decreases with increasing $\beta_{\mathrm{r}, \mathrm{eff}, \mathrm{d}, \mathrm{i}} / \beta_{\mathrm{r}, \mathrm{eff}, \mathrm{s}, \mathrm{i}}$.

The parameter, $\xi_{\mathrm{d}, \mathrm{i}} / \xi_{\mathrm{s}, \mathrm{i}}$, represents the ratio of the cumulative effects of the reaction stoichiometry and density of the copper colony to those of the surrounding silicon. The decorating efficiency $I$ increases with increasing $\xi_{\mathrm{d}, \mathrm{i}} / \xi_{\mathrm{s}, \mathrm{i}}$, for a fixed $R_{\mathrm{r}, \mathrm{eff}, \mathrm{d}, \mathrm{i}} / R_{\mathrm{r}, \mathrm{eff}, \mathrm{s}, \mathrm{i}}$. Figure $3 \mathrm{~b}$ shows the dependence of the decorating efficiency at the microdefect site, on $\beta_{\mathrm{m}, \mathrm{eff}, \mathrm{d}, \mathrm{i}} / \beta_{\mathrm{r}, \text { eff,d,i }}$, for different $\xi_{\mathrm{d}, \mathrm{i}} / \xi_{\mathrm{s}, \mathrm{i}}$, at a fixed $R_{\mathrm{r}, \text { eff,d,i }} / R_{\mathrm{r}, \mathrm{eff}, \mathrm{s}, \mathrm{i}}$. Each line on this graph represents a constant $\xi_{\mathrm{d}, \mathrm{i}} / \xi_{\mathrm{s}, \mathrm{i}}$

The corresponding polishing behavior of any etchant is studied on a graph of microdefect site polishing efficiency $v s$. $\beta_{\mathrm{m}, \text { eff,d,i }} / \beta_{\mathrm{r}, \mathrm{eff}, \mathrm{d}, \mathrm{i}}$ (Fig. 4a). Clearly, the polishing capacity and the microdefect-decorating capacity exhibit an inverse relationship with each other. Microdefect-polishing tendency increases with increasing $\beta_{\mathrm{r}, \mathrm{eff}, \mathrm{d}, \mathrm{i}} / \beta_{\mathrm{r}, \text { eff,s,i }}$. Again, Fig. 4a shows the isokinetic lines when $\xi_{\mathrm{d}, \mathrm{i}} / \xi_{\mathrm{s}, \mathrm{i}}$ is unity. There is a family of isokinetic lines for each

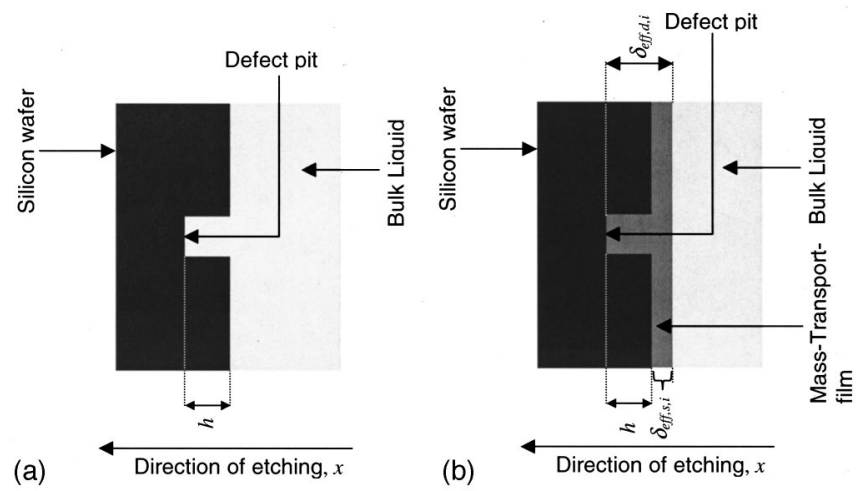

Figure 5. A phenomenological representation of microdefect decoration for a finite mass-transport film thickness and (a) infinite effective diffusivity, (b) finite effective diffusivity. $\xi_{\mathrm{d}, \mathrm{i}} / \xi_{\mathrm{s}, \mathrm{i}}$. Figure $4 \mathrm{~b}$ shows constant $\xi_{\mathrm{d}, \mathrm{i}} / \xi_{\mathrm{s}, \mathrm{i}}$ for a given $R_{\mathrm{r}, \text { eff, d,i }} / R_{\mathrm{r}, \text { eff,s,i }}$. The polishing efficiency decreases with increasing $\xi_{\mathrm{d}, \mathrm{i}} / \xi_{\mathrm{s}, \mathrm{i}}$.

Limiting case 2: Effect of diffusivity.-The mass-transport time scale decreases with increasing diffusivity. Thus, both polishing efficiency and decorating efficiency at the microdefect site are affected by the diffusivities of reactants for a fixed transport-film thickness. Consider the hypothetical scenario shown in Fig. 5. Figure 5a depicts the case for infinite diffusivity. When the diffusivity is infinite the thickness of the mass-transport film is irrelevant as the masstransport resistance is zero. Under these conditions, the decorating efficiency is at its highest (maximum) value and the polishing efficiency is at its lowest (minimum) value. Equations 18 and 19 quantify these efficiencies. When the diffusivity of the key reactant decreases (Fig. 5b), the decorating efficiency decreases and the polishing efficiency increases. For these conditions, the decorating and polishing efficiencies are quantified by Eq. 12 and 16, respectively. As the diffusivity of the key reactant further decreases, the crossover occurs when the sum of the kinetic and the transport resistances at the etch pit at the microdefect site equals the sum of the kinetic and the transport resistances on the perfect silicon surface

$$
\beta_{\mathrm{r}, \mathrm{eff}, \mathrm{d}, \mathrm{i}}+\beta_{\mathrm{m}, \mathrm{eff}, \mathrm{d}, \mathrm{i}}-\left(\beta_{\mathrm{r}, \mathrm{eff}, \mathrm{s}, \mathrm{i}}+\beta_{\mathrm{m}, \mathrm{eff}, \mathrm{s}, \mathrm{i}}\right)=0
$$

As diffusivity approaches zero, the decorating and microdefectpolishing efficiencies tend to the nonzero asymptotic values described by Eq. 26 and 27, respectively

$$
\begin{gathered}
\lim _{\mathrm{D}_{\mathrm{eff}, \mathrm{i}} \rightarrow 0} I=1-\frac{\xi_{\mathrm{s}, \mathrm{i}}}{\xi_{\mathrm{d}, \mathrm{i}}}\left(\frac{h}{\delta_{\mathrm{eff}, \mathrm{s}, \mathrm{i}}}+1\right) \\
\lim _{\mathrm{D}_{\mathrm{eff}, \mathrm{i}} \rightarrow 0} \eta=1-\frac{\xi_{\mathrm{d}, \mathrm{i}}}{\xi_{\mathrm{s}, \mathrm{i}}} \frac{\delta_{\mathrm{eff}, \mathrm{s}, \mathrm{i}}}{\delta_{\mathrm{eff}, \mathrm{s}, \mathrm{i}}+h}
\end{gathered}
$$

It is quite interesting to note that for $\xi_{\mathrm{d}, \mathrm{i}} / \xi_{\mathrm{s}, \mathrm{i}}=1$, the asymptotic value of the decorating efficiency at the etch pit at the microdefect site is a constant negative value and the asymptotic value of the polishing efficiency at the site is a constant positive value. Thus, when the thickness of the transport film is finite, increase in the transport resistance invariably improves the polishing quality and deteriorates the decorating capacity of an etchant.

The effects of transport and kinetics on microdefect decoration can be illustrated on a graph of decorating efficiency $(I) v s$. the ratio of mass-transport resistance to the kinetic resistance $\left(\beta_{\mathrm{m}, \mathrm{eff}, \mathrm{d}, \mathrm{i}} / \beta_{\mathrm{r}, \mathrm{eff}, \mathrm{d}, \mathrm{i}}\right)$. Figure $6 \mathrm{a}$ shows isokinetic lines on this graph for $\xi_{\mathrm{d}, \mathrm{i}} / \xi_{\mathrm{s}, \mathrm{i}}=1$. As discussed earlier, an isokinetic line represents a fixed kinetics $\left(\beta_{\mathrm{r}, \mathrm{eff}, \mathrm{d}, \mathrm{i}} / \beta_{\mathrm{r}, \text { eff,s,i }}\right)$. The decorating efficiency decreases with increasing $\beta_{\mathrm{r}, \mathrm{eff}, \mathrm{d}, \mathrm{i}} / \beta_{\mathrm{r}, \mathrm{eff}, \mathrm{s}, \mathrm{i}}$. Figure $6 \mathrm{~b}$ shows the effect of $\xi_{\mathrm{d}, \mathrm{i}} / \xi_{\mathrm{s}, \mathrm{i}}$ at a given $R_{\mathrm{r}, \mathrm{eff}, \mathrm{d}, \mathrm{i}} / R_{\mathrm{r}, \mathrm{eff}, \mathrm{s}, \mathrm{i}}$. Here decorating efficiency increases with increasing $\xi_{\mathrm{d}, \mathrm{i}} / \xi_{\mathrm{s}, \mathrm{i}}$.
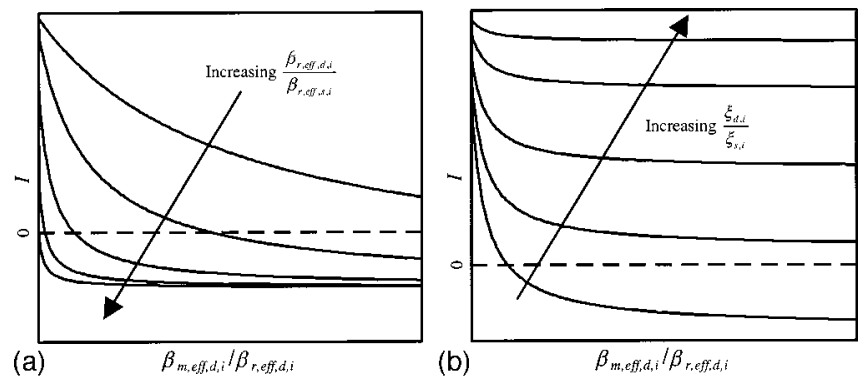

Figure 6. Effect of the effective diffusivity on the decorating efficiency at a microdefect site for a given pit depth, mass-transport film thickness, and (a) $\xi_{\mathrm{d}, \mathrm{i}} / \xi_{\mathrm{s}, \mathrm{i}},(\mathrm{b}) R_{\mathrm{r}, \mathrm{eff}, \mathrm{d}, \mathrm{i}} / R_{\mathrm{r}, \mathrm{eff}, \mathrm{s}, \mathrm{i}}$. 


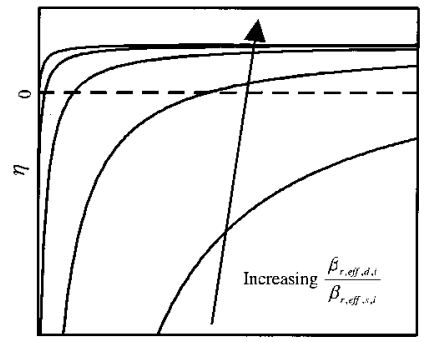

(a)

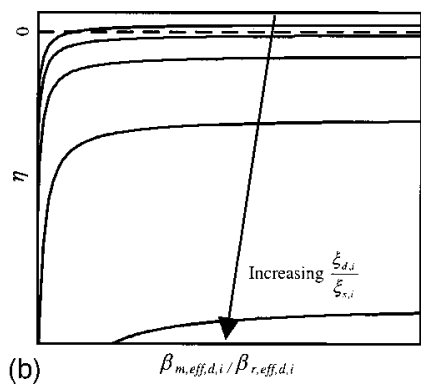

(b) tate colony. The pit depth is at its maximum when the decorating efficiency becomes zero. This condition is quantified by Eq. 30

$$
\begin{aligned}
h_{\mathrm{max}} & =\frac{\xi_{\mathrm{d}, \mathrm{i}}}{\xi_{\mathrm{s}, \mathrm{i}}} \delta_{\text {eff, } \mathrm{s}, \mathrm{i}}\left[\left(\frac{\beta_{\mathrm{r}, \mathrm{eff}, \mathrm{s}, \mathrm{i}}-\beta_{\mathrm{r}, \mathrm{eff}, \mathrm{d}, \mathrm{i}}}{\beta_{\mathrm{m}, \mathrm{eff}, \mathrm{s}, \mathrm{i}}}\right)-\left(\frac{\xi_{\mathrm{s}, \mathrm{i}}}{\xi_{\mathrm{d}, \mathrm{i}}}-1\right)\right] \\
& =\xi_{\mathrm{d}, \mathrm{i}} D_{\mathrm{eff}, \mathrm{i}}\left(\beta_{\mathrm{r}, \mathrm{eff}, \mathrm{s}, \mathrm{i}}-\beta_{\mathrm{r}, \mathrm{eff}, \mathrm{d}, \mathrm{i}}\right)-\delta_{\mathrm{eff}, \mathrm{s}, \mathrm{i}}\left(1-\frac{\xi_{\mathrm{d}, \mathrm{i}}}{\xi_{\mathrm{s}, \mathrm{i}}}\right)
\end{aligned}
$$

Figure 7. Effect of the effective diffusivity on the polishing efficiency at (a) a given microdefect site for a given pit depth, mass-transport film thickness, and $\xi_{\mathrm{d}, \mathrm{i}} / \xi_{\mathrm{s}, \mathrm{i}}$, (b) a microdefect site for a given pit depth, mass-transport film thickness, and $R_{\mathrm{r}, \text { eff,d,i }} / R_{\mathrm{r}, \mathrm{eff}, \mathrm{s}, \mathrm{i}}$.

The corresponding polishing behavior when $\xi_{\mathrm{d}, \mathrm{i}} / \xi_{\mathrm{s}, \mathrm{i}}$ is unity is shown in Fig. 7a. The polishing efficiency at an etch pit at a microdefect site increases with increasing $\beta_{\mathrm{m}, \mathrm{eff}, \mathrm{d}, \mathrm{i}} / \beta_{\mathrm{r}, \text { eff,d,i }}$ and reaches an asymptotic positive value. It is also shown that the polishing efficiency increases with increasing $\beta_{\mathrm{m}, \text { eff,d,i }} / \beta_{\mathrm{r}, \text { eff,s,i }}$. The effect of $\xi_{\mathrm{d}, \mathrm{i}} / \xi_{\mathrm{s}, \mathrm{i}}$ on the polishing efficiency is shown in Fig. 7b. The polishing efficiency decreases with increasing $\xi_{\mathrm{d}, \mathrm{i}} / \xi_{\mathrm{s}, \mathrm{i}}$.

\section{Autoinhibiting Decoration}

Weak microdefect decoration in the presence of the masstransport effects can be autoinhibiting in nature. The etch-pit depth (h) at a microdefect site increases for a positive local decorating efficiency. However, as the pit depth increases, the effective masstransport resistance on the pit increases while the effective masstransport resistance on the perfect silicon surface remains relatively unchanged. Therefore, the decorating efficiency gradually decreases with increasing pit depth. This autoinhibition is explained by Eq. 12 and 16 , which can be rewritten as

$$
\begin{gathered}
I=1-\frac{\beta_{\mathrm{r}, \mathrm{eff}, \mathrm{d}, \mathrm{i}}}{\beta_{\mathrm{r}, \mathrm{eff}, \mathrm{s}, \mathrm{i}}}\left[\frac{1+\left(\frac{\left(h+\delta_{\mathrm{eff}, \mathrm{s}, \mathrm{i}}\right)}{D_{\mathrm{eff}, \mathrm{i}}} \frac{1}{\xi_{\mathrm{d}, \mathrm{i}} \beta_{\mathrm{r}, \mathrm{eff}, \mathrm{d}, \mathrm{i}}}\right)}{1+\left(\frac{\delta_{\mathrm{eff}, \mathrm{i}, \mathrm{i}}}{D_{\mathrm{eff}, \mathrm{i}}} \frac{1}{\xi_{\mathrm{s}, \mathrm{i}} \beta_{\mathrm{r}, \mathrm{eff}, \mathrm{s}, \mathrm{i}}}\right)}\right] \\
\eta=1-\frac{\beta_{\mathrm{r}, \mathrm{eff}, \mathrm{s}, \mathrm{i}}}{\beta_{\mathrm{r}, \mathrm{eff}, \mathrm{d}, \mathrm{i}}}\left[\frac{1+\left(\frac{\delta_{\mathrm{eff}, \mathrm{s}, \mathrm{i}}}{D_{\mathrm{eff}, \mathrm{i}}} \frac{1}{\xi_{\mathrm{s}, \mathrm{i}} \beta_{\mathrm{r}, \mathrm{eff}, \mathrm{s}, \mathrm{i}}}\right)}{1+\left(\frac{\left(h+\delta_{\mathrm{eff}, \mathrm{s}, \mathrm{i}}\right)}{D_{\mathrm{eff}, \mathrm{i}}} \frac{1}{\xi_{\mathrm{d}, \mathrm{i}} \beta_{\mathrm{r}, \mathrm{eff}, \mathrm{d}, \mathrm{i}}}\right)}\right]
\end{gathered}
$$

Thus, the intensity of microdefect decoration decreases as the decoration proceeds. For an etchant sensitive to increase in the film thickness, the local polishing efficiency at the etch pit can become positive even before the complete dissolution of the copper precipi-
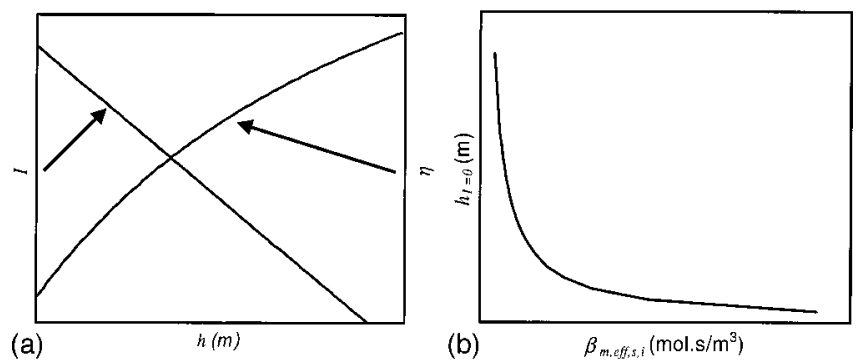

Figure 8. Auto inhibition of microdefect decoration (a) with increasing pit depth, (b) as a function of the mass-transport resistance.

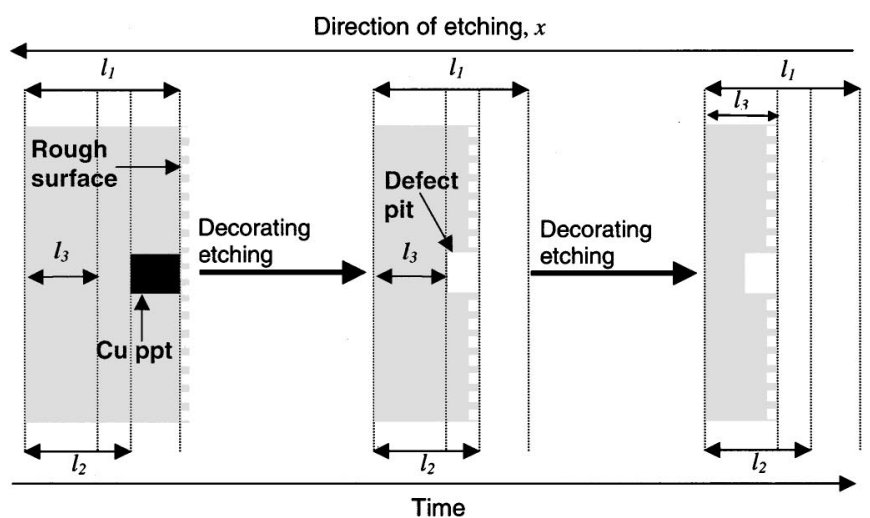

(a)

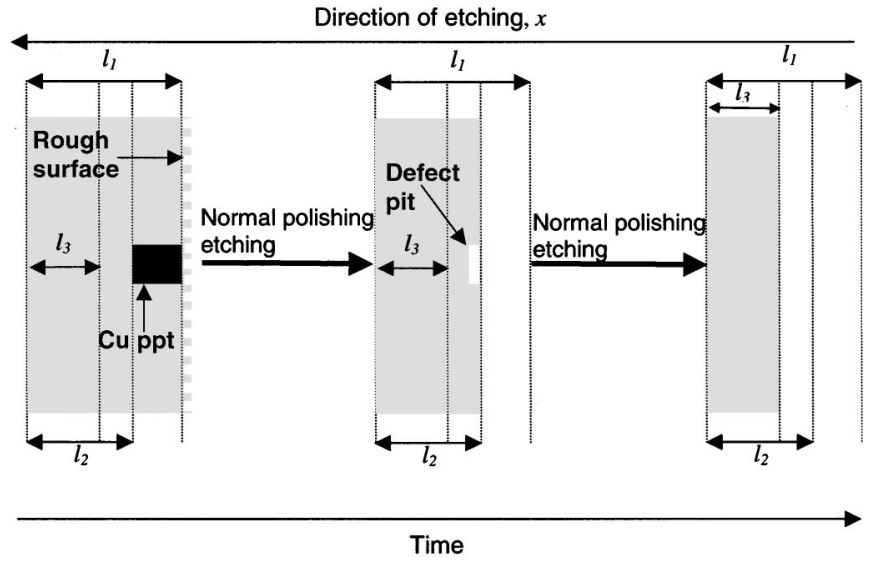

(b)

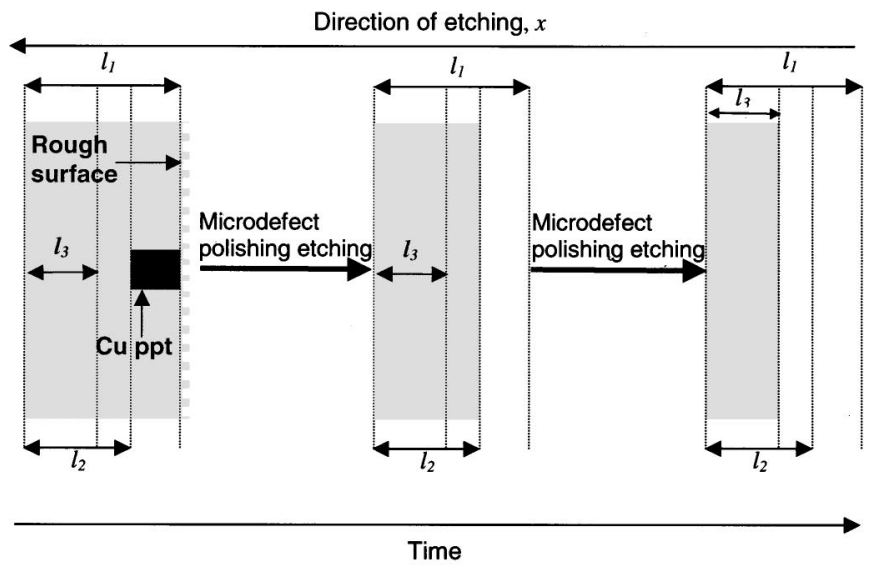

(c)

Figure 9. A schematic representation of (a) microdefect decoration by a decorating etchant, (b) a weak microdefect decoration and subsequent erosion by a normal polishing etchant, (c) microdefect erosion by a microdefectpolishing etchant. 


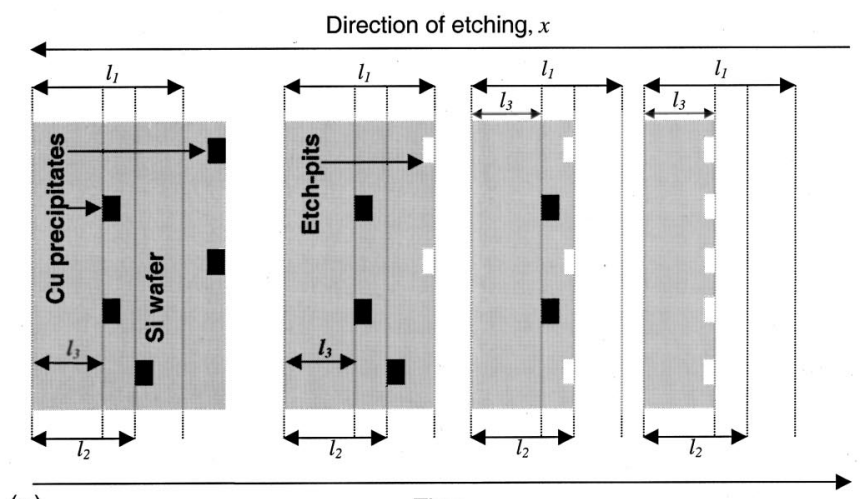

(a)

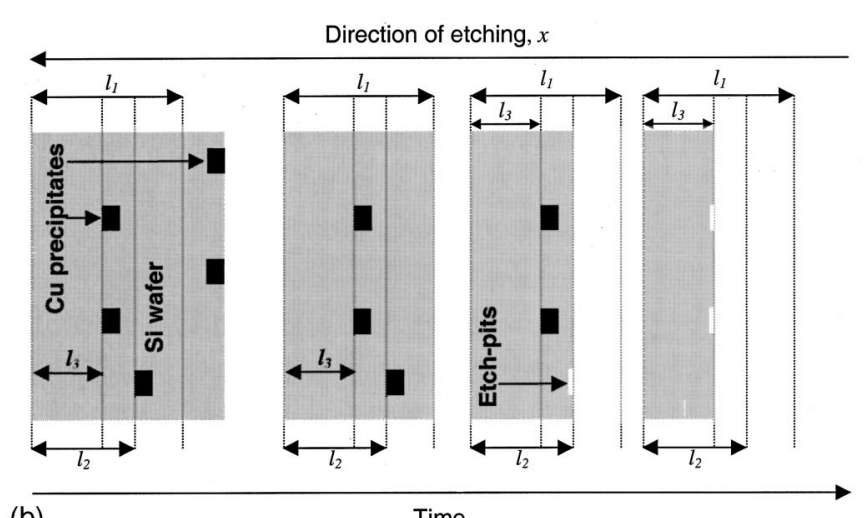

(b)

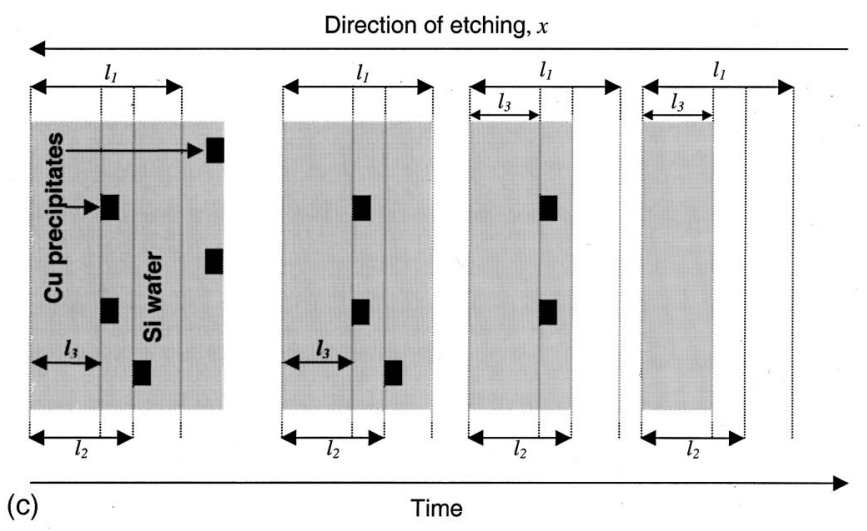

Figure 10. A global picture of (a) microdefect decoration by a decorating etchant, (b) weak microdefect decoration and subsequent erosion by a normal polishing etchant, (c) microdefect erosion by a microdefect-polishing etchant. where the subscript max denotes the maximum value. The autoinhibiting capacity increases with decreasing effective diffusivity, $D_{\mathrm{i}, \text { eff }}$. Autoinhibition can be described on a graph of local decorating efficiency vs. pit depth, as shown in Fig. 8a. Autoinhibition shows an inverse relationship with effective diffusivity. It is clear that etchants with higher effective mass-transport resistances show greater tendencies toward autoinhibition. In other words, microdefect decoration is very poor when the transport effects are dominant. Figure $8 \mathrm{~b}$ shows the effect of the mass transport on autoinhibition on an $h_{\text {max }} v s . \beta_{\text {m,eff,s,i }}$ graph.

\section{Theoretical Classification of Etchants}

In the following sections, decorating and polishing capacities of various etchants for precipitate-enhanced microdefects are discussed. However, this discussion can be extended to native microdefects in the absence of impurity precipitation. In this study, all experiments for microdefect decoration were accomplished using copper precipitation. Hence, the terms microdefect decoration and microdefect polishing generally refer to decoration and polishing, respectively, in the presence of the copper precipitate colonies.

The quantitative analysis of the polishing and the decorating capacity of an etchant leads to its classification. Within the scope of this study, an etchant can be classified in four categories: neutral, decorating, microdefect polishing, and normal polishing. A neutral etchant shows negligible decorating and polishing efficiencies in the presence of a precipitate colony. A decorating etchant shows a positive decorating efficiency in the presence of a precipitate colony. A microdefect-polishing etchant shows a positive polishing efficiency at a microdefect site in the presence of a precipitate colony. Microdefect decoration is completely inhibited in the presence of a microdefect-polishing etchant. A normal polishing etchant shows a positive decorating efficiency at a site in the presence of a precipitate colony and a positive polishing efficiency at the same site upon dissolution of the colony. This happens because the ratio of kinetic resistance at the microdefect site to that on the perfect silicon surface $\left(\beta_{\mathrm{r}, \mathrm{eff}, \mathrm{d}, \mathrm{i}} / \beta_{\mathrm{r}, \mathrm{eff}, \mathrm{s}, \mathrm{i}}\right)$ is greater in the absence of precipitate colonies, than that in the presence of these colonies. Thus, a normal polishing etchant can decorate microdefects weakly by creating etch pits in the presence of precipitate colonies, and subsequently, polish and erase these etch pits after complete dissolution of the colonies. In a copper precipitated wafer, it is normal to have local variation of the polishing efficiency of an etchant, positive at the perfect region away from the copper precipitate colonies, and negative at the microdefect sites in the presence of the colonies. It is evident that in the absence of the copper precipitate colonies, both normal polishing and microdefect polishing etchants decrease surface irregularities such as surface roughness, whereas, both the neutral and decorating etchants cannot do so. Figure 9 describes the working models for the decorating, normal polishing, and microdefect-polishing etchants.

Both microdefect-polishing and normal polishing etchants can be classified as polishing etchants. A polishing etchant is sensitive to hydrodynamic conditions in the etching reactor, whereas a decorating etchant is relatively independent of the hydrodynamic conditions. The transport-film thickness decreases with increasing mixing

Table I. Components of various popular etchants.

\begin{tabular}{|c|c|c|c|c|}
\hline Etchant & Oxidant & Dissolving agent & Diluent & Classification \\
\hline $\mathrm{MAE}^{\mathrm{a}}$ & $\mathrm{HNO}_{3}$ & $\mathrm{HF}$ & $\mathrm{CH}_{3} \mathrm{COOH}+\mathrm{H}_{2} \mathrm{O}$ & Polishing \\
\hline $\mathrm{HF}+\mathrm{HNO}_{3}{ }^{\mathrm{a}}$ & $\mathrm{HNO}_{3}$ & $\mathrm{HF}$ & $\mathrm{H}_{2} \mathrm{O}$ & Polishing \\
\hline $\mathrm{HF}+\mathrm{HNO}_{3}+\mathrm{H}_{3} \mathrm{PO}_{4}{ }^{\mathrm{a}}$ & $\mathrm{HNO}_{3}$ & $\mathrm{HF}$ & $\mathrm{H}_{3} \mathrm{PO}_{4}+\mathrm{H}_{2} \mathrm{O}$ & Polishing \\
\hline $\mathrm{Secco}^{\mathrm{a}}$ & $\mathrm{K}_{2} \mathrm{Cr}_{2} \mathrm{O}_{7}$ & $\mathrm{HF}$ & $\mathrm{H}_{2} \mathrm{O}$ & Decorating \\
\hline Wright $^{\mathrm{a}}$ & $\mathrm{K}_{2} \mathrm{Cr}_{2} \mathrm{O}_{7}+\mathrm{HNO}_{3}+\mathrm{Cu}\left(\mathrm{NO}_{3}\right)_{2}$ & $\mathrm{HF}$ & $\mathrm{CH}_{3} \mathrm{COOH}+\mathrm{H}_{2} \mathrm{O}$ & Decorating \\
\hline
\end{tabular}

\footnotetext{
actual concentrations of the components may be different from what is reported in the literature.
} 


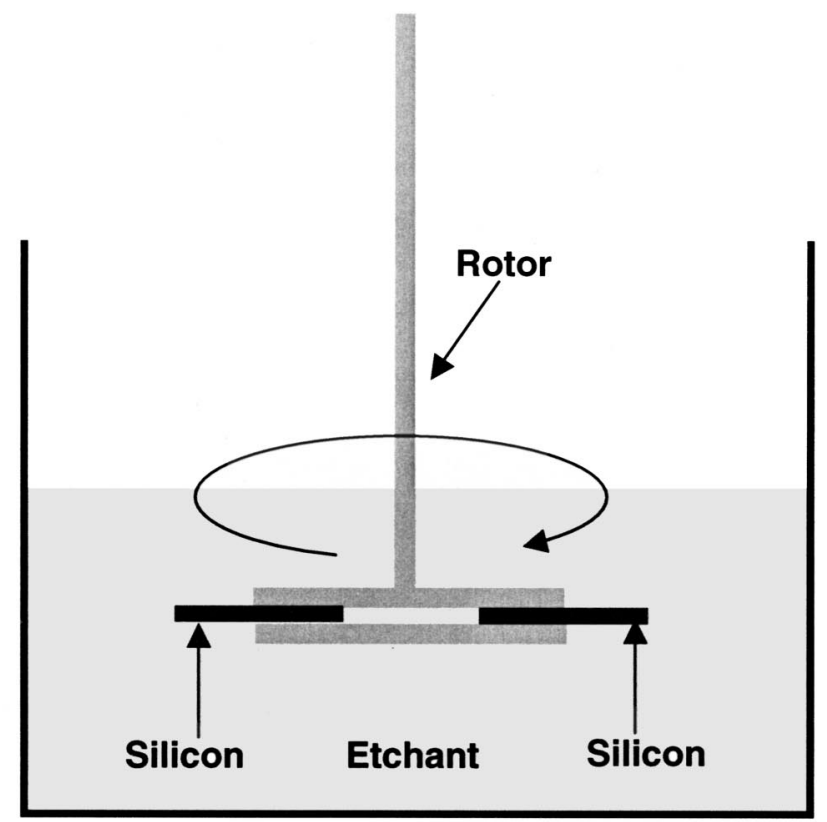

(a)

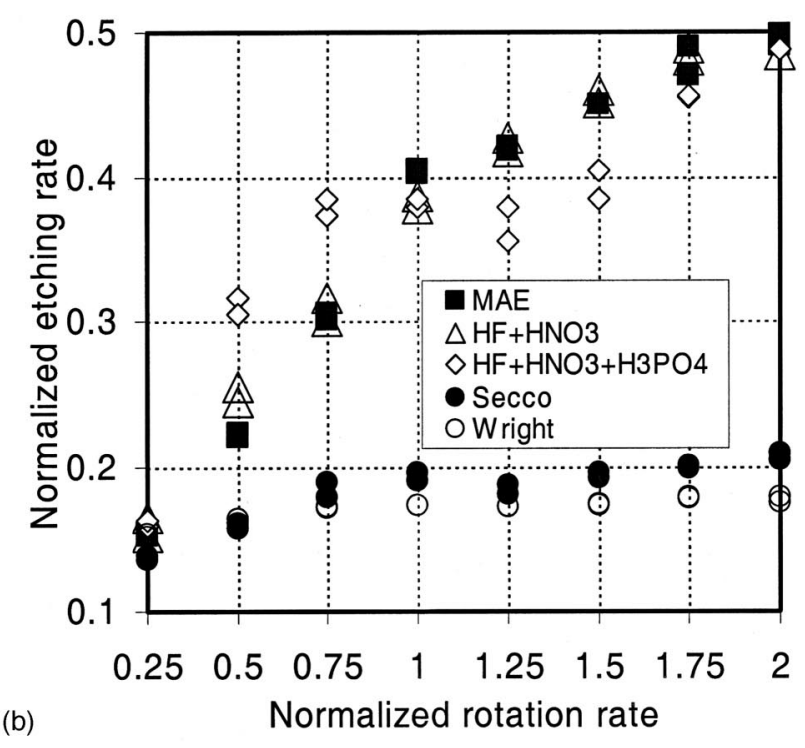

Figure 11. (a) An experimental assembly to etch silicon at different controlled mixing intensities. (b) The dependence of etching rate as a function of the reactor mixing intensity for various etchants.

intensity in a mass-transport influenced reactor. ${ }^{38,39}$ Thus, etching rate increases with mixing intensity under polishing conditions as a result of decreasing mass-transport film thickness. Conversely, etching rate does not show a significant change with mixing intensity under decorating conditions, because the mass-transport film is practically absent. However, note that neutral etchants can also show insensitivity to the hydrodynamic conditions. Only those etchants that are relatively independent of the hydrodynamic conditions and show a difference between the microdefect kinetics and the surface kinetics are decorating. Therefore, the etchants that are insensitive to mixing intensity can be defined as potentially decorating at best rather than decorating.

Thus, etchants can be divided into two broader categories, potentially decorating and polishing. The potentially decorating etchants include both decorating etchants and neutral etchants. The polishing
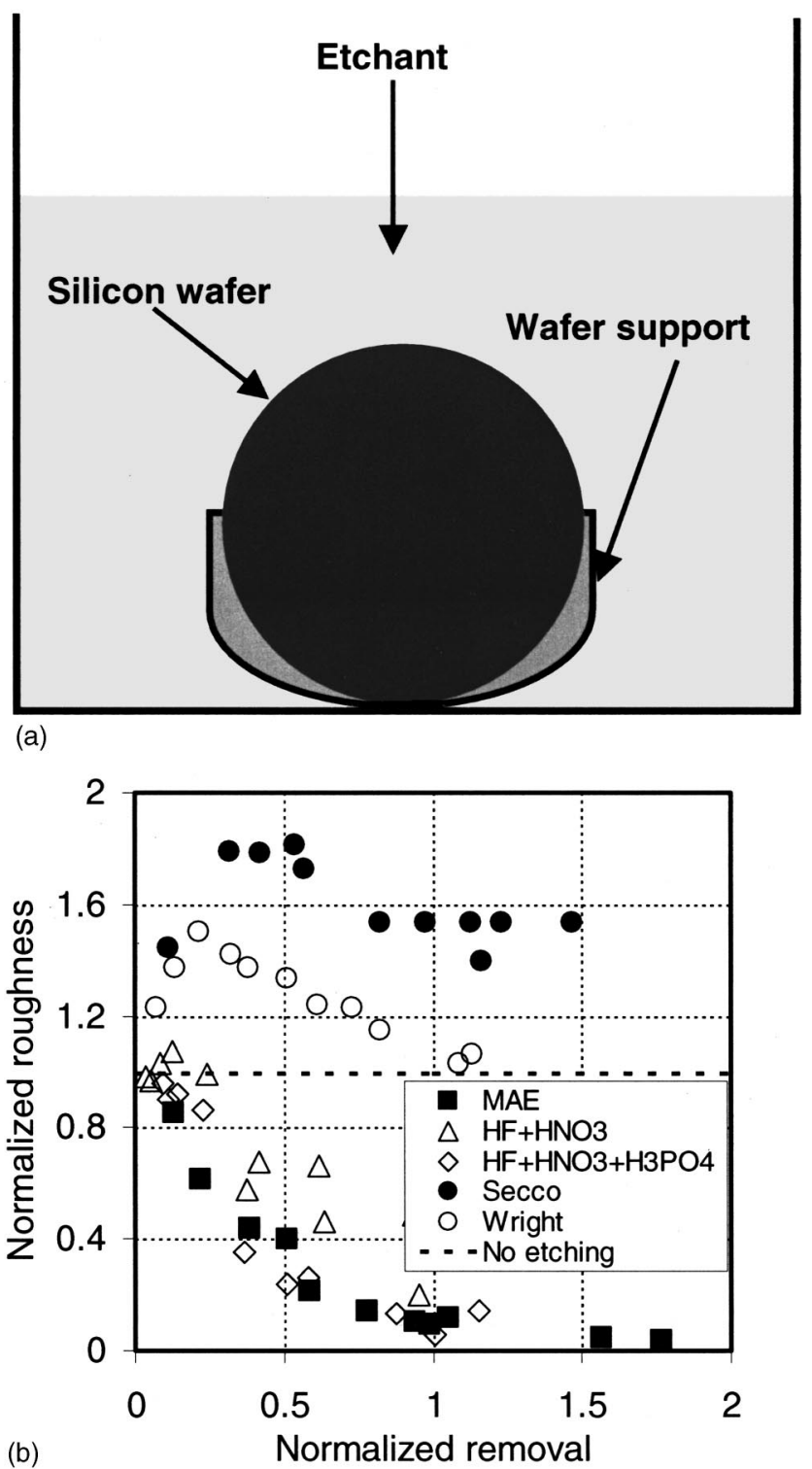

Figure 12. (a) An experimental assembly to etch silicon wafers in a given etchant. (b) The polishing effect of various popular etchants.

etchants include both normal polishing and microdefect-polishing etchants.

\section{Microdefect Decoration}

A global picture.-The total number of etch pits on a decorated silicon wafer is a function of the microdefect density and the total removal. Figure 10a schematically shows the global mechanism of microdefect decoration in the presence of a decorating etchant. The copper precipitate colonies are of finite dimension. A copper precipitate colony dissolves into the etching mixture by creating a pit, which becomes an irregular perfect silicon site upon complete dissolution of the colony. Since the decorating etchant lacks the surface polishing quality, the pits formed on the surface maintain their identity throughout the etching process while interior copper precipitate colonies are exposed as new pits. Thus, the apparent surface density of the pits is a function of the true microdefect density and the total linear removal by the decorating etchant.

A normal polishing etchant weakly decorates the microdefects by creating the etch pits and eventually erases them. Thus, microdefect decoration by a normal polishing etchant is sparse and very weak. 


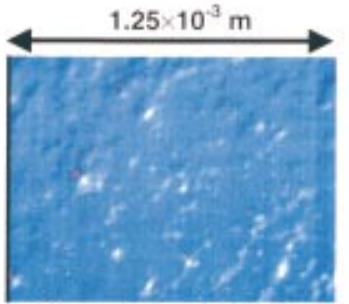

Microphoto: A defects

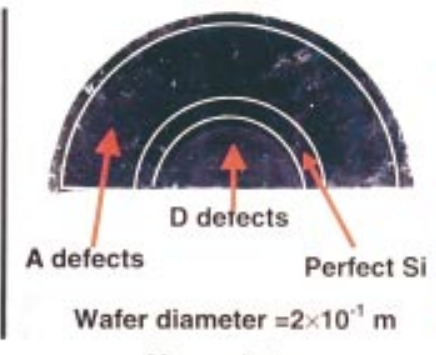

Macrophoto

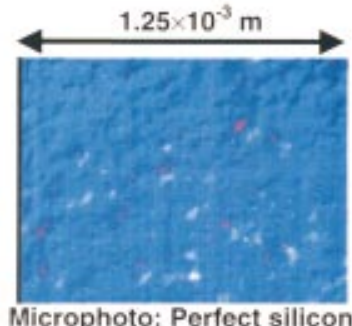

Microphoto: Perfect silicon

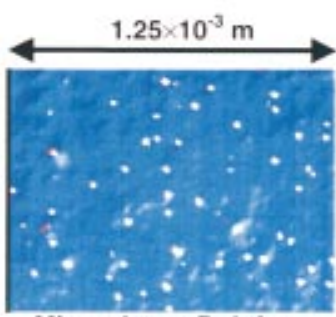

Microphoto: D defects
Figure 13. Microdefect related etch pits in a reference wafer after copper precipitation and surface polishing.

Shallow pits are formed by this etchant at the microdefect sites exposed at the end of etching. Figure 10b shows weak microdefect decoration by a normal polishing etchant. The microdefect-polishing etchant does not allow etch-pit formation, and hence, there is no defect decoration (Fig. 10c).

\section{Experimental Classification of Etchants}

The theoretical analysis proposed in the previous sections was experimentally verified. A group of decorating and polishing etchants was chosen. Table I lists the components of the chosen etchants. Note that actual concentrations of the components play a

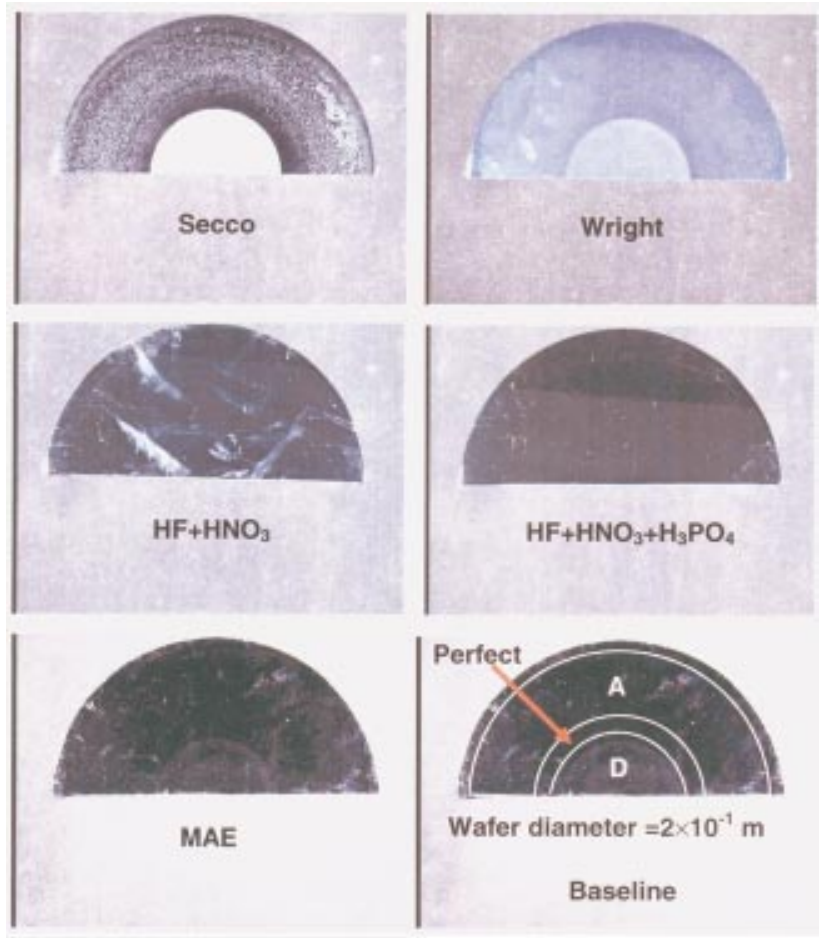

Figure 14. Macrophotos of microdefect decoration by different etchants.

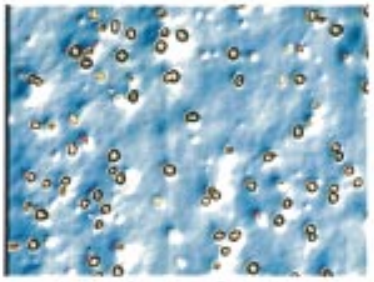

D defects: Secco

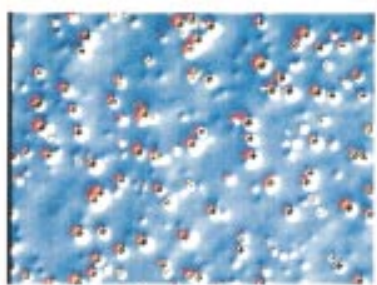

D defects: Wright

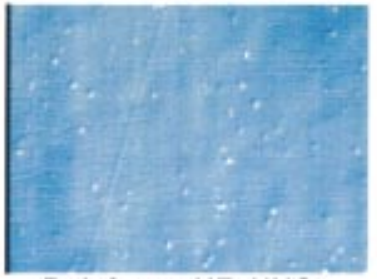

D defects: $\mathrm{HF}+\mathrm{HNO}_{3}$

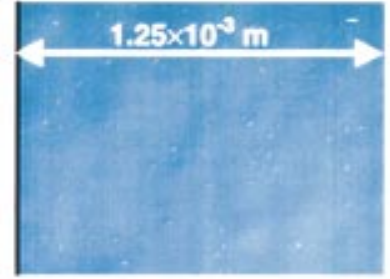

D defects: $\mathrm{HF}+\mathrm{HNO}_{3}+\mathrm{H}_{3} \mathrm{PO}_{4}$

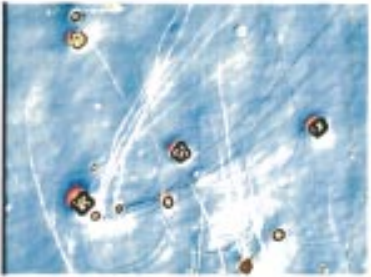

A defects: Secco

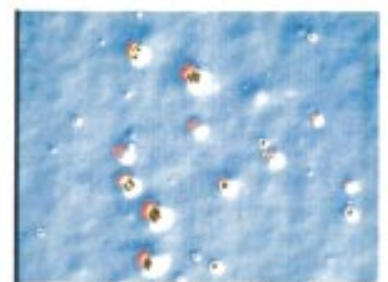

A defects: Wright

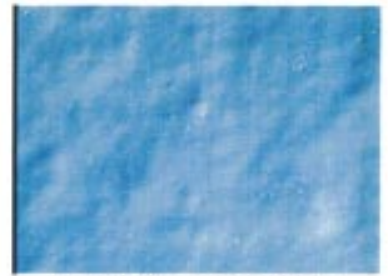

A defects: $\mathrm{HF}+\mathrm{HNO}_{3}$

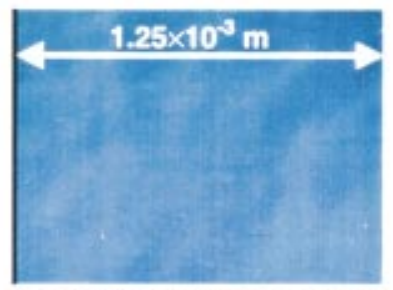

A defects: $\mathrm{HF}+\mathrm{HNO}_{3}+\mathrm{H}_{3} \mathrm{PO}_{4}$
Figure 15. Microphotos of microdefect decoration by different etchants.

very important role in defining the nature of an etchant. However, it is not necessary to reveal the actual concentrations of the components for our study. According to the theory of etching discussed in this study, the potentially decorating and polishing etchants show distinct hydrodynamic responses. The classification of the chosen etchants into two groups, namely, potentially decorating and polishing, is accomplished by doing two sets of experiments.

First, precipitate-free silicon pieces were etched in a given etchant in the experimental assembly shown in Fig. 11a. The assembly includes a mechanism that allows the etching of silicon pieces at different rotation rates. The rotation rate is a reasonable measure of the mixing intensity in the etching tank. Using this experimental assembly the etching rate $v s$. rotation rate data is collected for all etchants (Fig. 11b). Secco and Wright etchants show a weak dependence on the mixing intensity whereas mixed acid etchant (MAE), $\mathrm{HF}+\mathrm{HNO}_{3}$, and $\mathrm{HF}+\mathrm{HNO}_{3}+\mathrm{H}_{3} \mathrm{PO}_{4}$ show a significant increase in etching rates with increasing mixing intensity. At this stage, it can be concluded that Secco and Wright are potentially decorating etchants, and $\mathrm{MAE}, \mathrm{HF}+\mathrm{HNO}_{3}$, and $\mathrm{HF}+\mathrm{HNO}_{3}$ $+\mathrm{H}_{3} \mathrm{PO}_{4}$ are polishing etchants.

Second, precipitate-free silicon wafers were etched by different etchants in an etchant bath as shown in Fig. 12a. The same hydrodynamic conditions were maintained for all etchants. Change in surface roughness as a function of linear removal was recorded for each etchant (Fig. 12b). The polishing etchants decrease the surface 


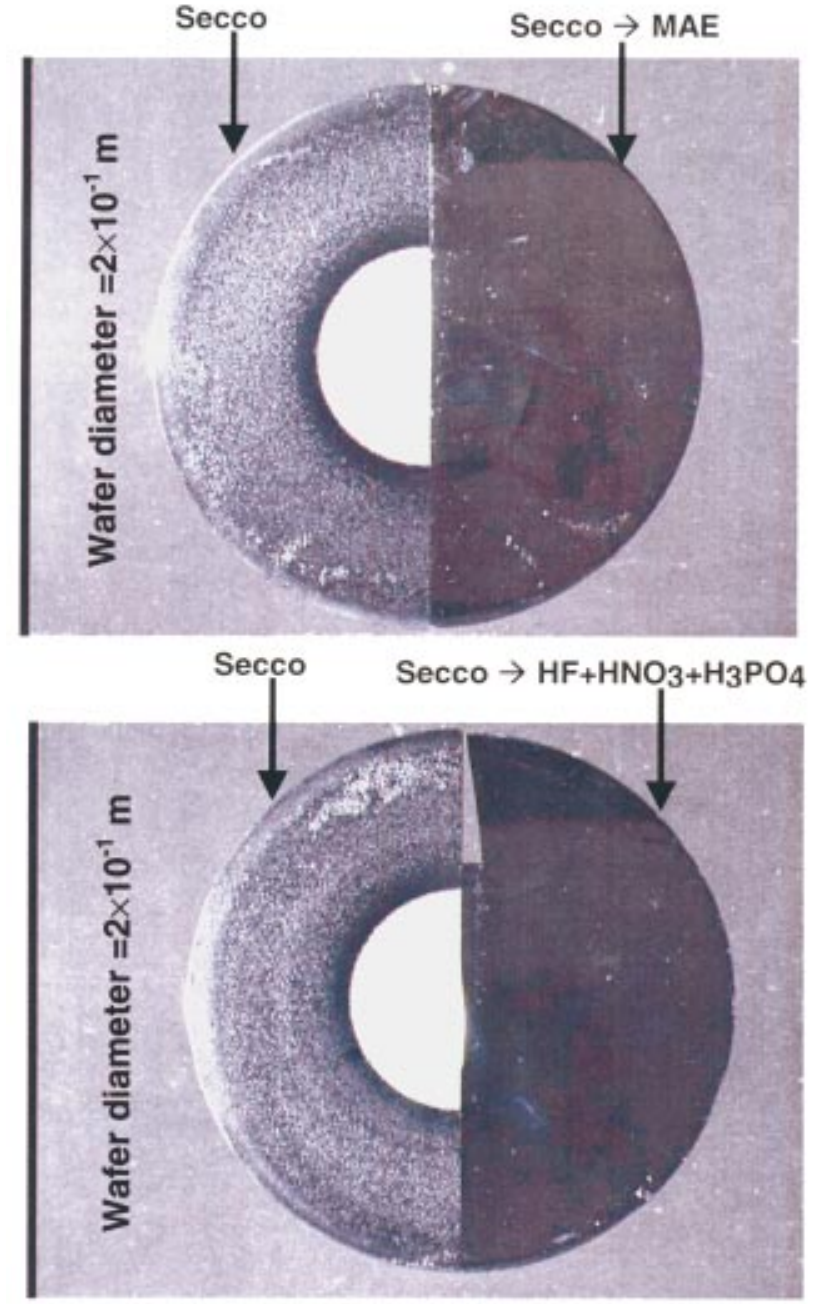

Figure 16. Erosion of the microdefect related etch pits by polishing etchants: Macrophoto.

roughness with increasing removal, whereas potentially decorating etchants do not decrease the surface roughness. ${ }^{36}$ This experiment clearly establishes MAE, $\mathrm{HF}+\mathrm{HNO}_{3}$, and $\mathrm{HF}+\mathrm{HNO}_{3}+\mathrm{H}_{3} \mathrm{PO}_{4}$ as polishing etchants, and Secco and Wright as potentially decorating etchants. The data points inside two ellipses in Fig. 12b indicate very high roughness readings beyond the sensitivity of the roughness gauge used. Nevertheless, in this case, the roughness does not decrease with removal, and hence, the argument holds.

\section{Normal Polishing and Potentially Decorating Etchants}

Experimental comparison.-Based on the proposed models, MAE, $\mathrm{HF}+\mathrm{HNO}_{3}$, and $\mathrm{HF}+\mathrm{HNO}_{3}+\mathrm{H}_{3} \mathrm{PO}_{4}$ are classified as polishing etchants, and Secco and Wright are potentially decorating etchants. Validity of the model can be verified by comparing the decorating capacity of the aforementioned etchants. The decorating performance of each etchant was experimentally verified by the standard procedure for microdefect decoration. Different silicon wafers having similar native microdefect distribution were used for all experiments discussed henceforth. First, silicon wafers were saturated with copper at $900^{\circ} \mathrm{C}$ and then cooled very quickly to room temperature. The fast cooling allows growth of copper precipitate colonies on the microdefects. During the cool-down, some excess copper also escapes to the wafer surface and forms tiny surface copper precipitate colonies. This wafer is then cleaned and polished

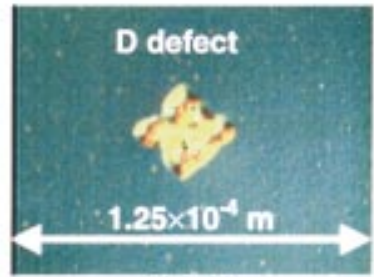

Wright

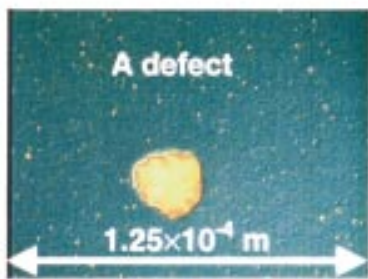

Wright

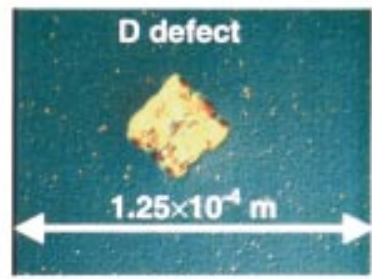

Wright

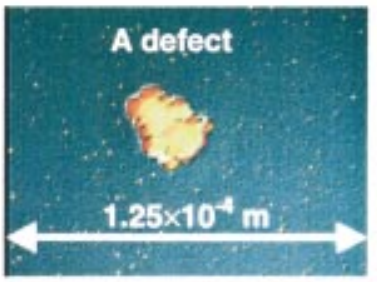

Wright

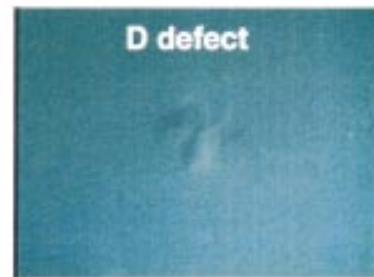

Wright $\rightarrow \mathrm{HF}+\mathrm{HNO}_{3}$

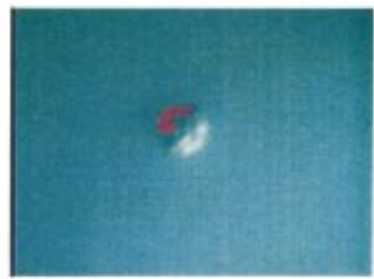

Wright $\rightarrow \mathrm{HF}+\mathrm{HNO}_{3}$

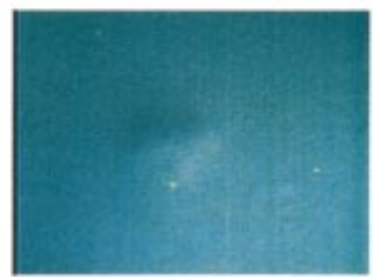

Wright $\rightarrow \mathrm{HF}+\mathrm{HNO}_{3}+\mathrm{H}_{3} \mathrm{PO}_{4}$

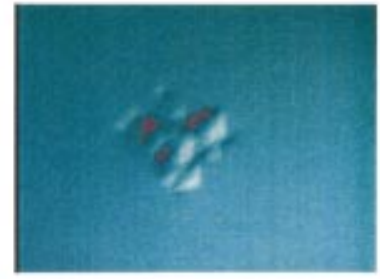

Wright $\rightarrow \mathrm{HF}+\mathrm{HNO}_{3}+\mathrm{H}_{3} \mathrm{PO}_{4}$
Figure 17. Erosion of the microdefect related etch pits by polishing etchants: Microphoto.

to remove the surface copper precipitate colonies. Surface polishing is accomplished by etching in any polishing etchant, such as MAE. Since the copper precipitate colonies exist only on the surface, even a normal polishing etchant would erase the surface etch pits formed by the surface copper precipitate colonies, given sufficient removal. The surface of a polish-etched MAE wafer is shown in Fig. 13. Since the microdefect patterns in a silicon wafer show azimuthal symmetry, only halves of any given wafer sample are used for these experiments. Figure 13 displays a digital image of the wafer visible to the naked eye, or macrophoto, and an image of the wafer as seen under a Nomarski microscope or microphoto. It is evident that A-defects are practically not decorated at all in the polishing step. However, a weak decoration of D-defects indicates that MAE acts as a normal polishing etchant in the D-defect region. Autoinhibition keeps the decoration very poor. The microdefect patterns shown in Fig. 13 form the basis for defining the performance of an etchant as either decorating or polishing.

The wafers subjected to copper precipitation and surface polishing were then etched by the different etchants under consideration. A typical result of microdefect decoration is shown in Fig. 14. It is evident that Secco and Wright are indeed decorating etchants, and MAE, $\mathrm{HF}+\mathrm{HNO}_{3}$, and $\mathrm{HF}+\mathrm{HNO}_{3}+\mathrm{H}_{3} \mathrm{PO}_{4}$ are at least normal 

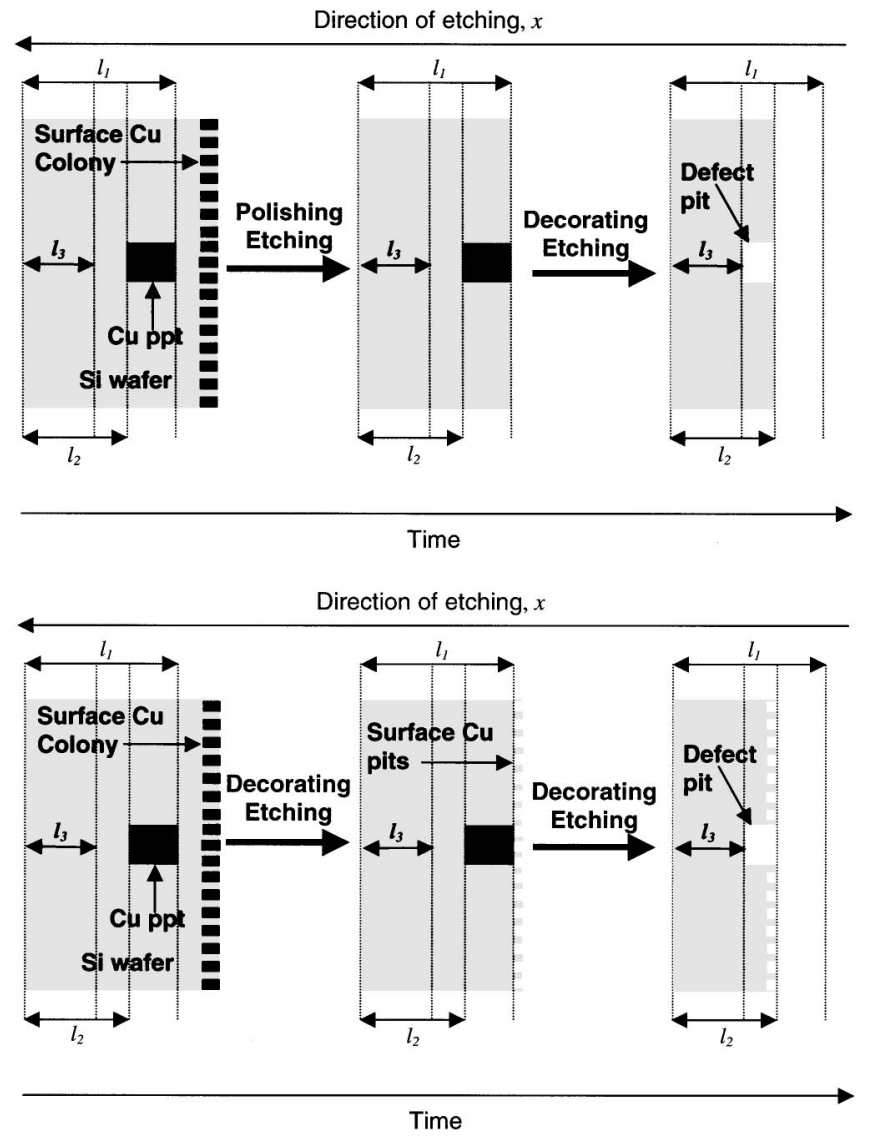

Figure 18. A schematic picture of (a) the standard microdefect decoration, (b) surface roughening in the absence of surface polishing.

polishing etchants. The microphotos of microdefect decoration (Fig. 15) further confirm this observation.

The behavior of different etchants under consideration is consistent with their classification as either potentially decorating or polishing, based on the kinetic and hydrodynamic responses. In addition, it can also be shown that a normal polishing etchant erases or at least reduces the depth of the etch pits created by a previous decoration by a decorating etchant. The following experiment of three sequential processes tests this hypothesis

\section{Copper precipitation $\rightarrow$ Decorating etching $\rightarrow$ Polishing etching}

The results of this experiment are shown in Fig. 16 and 17. Figure 16 displays the macrophotos of a wafer after Secco etching followed by MAE etching. Macrophotos of a wafer after Secco etching and subsequent $\mathrm{HF}+\mathrm{HNO}_{3}+\mathrm{H}_{3} \mathrm{PO}_{4}$ etching are also shown in Fig. 16. Similar sequential experiments were conducted with Wright and $\mathrm{HF}+\mathrm{HNO}_{3}$ etchants, and Wright and $\mathrm{HF}+\mathrm{HNO}_{3}+\mathrm{H}_{3} \mathrm{PO}_{4}$ etchants. The microphotos shown in Fig. 17 clearly indicate the reduction in the microdefect related etch-pit depth by polishing etchants. Hence, the hypothesis underlying the classification of the decorating and the polishing etchants based on the kinetic and the hydrodynamic time scales is verified.

Effect of surface copper precipitate colonies.-The proposed phenomenological model can be further verified by studying the effect of the copper precipitate colonies on the wafer surface. As explained before, during the wafer cool-down after its saturation with copper at a higher temperature, copper out-diffusion to the wafer surface takes place in addition to the $\mathrm{Cu}_{3} \mathrm{Si}$ precipitate formation on the microdefects. The out-diffused copper forms tiny pre-
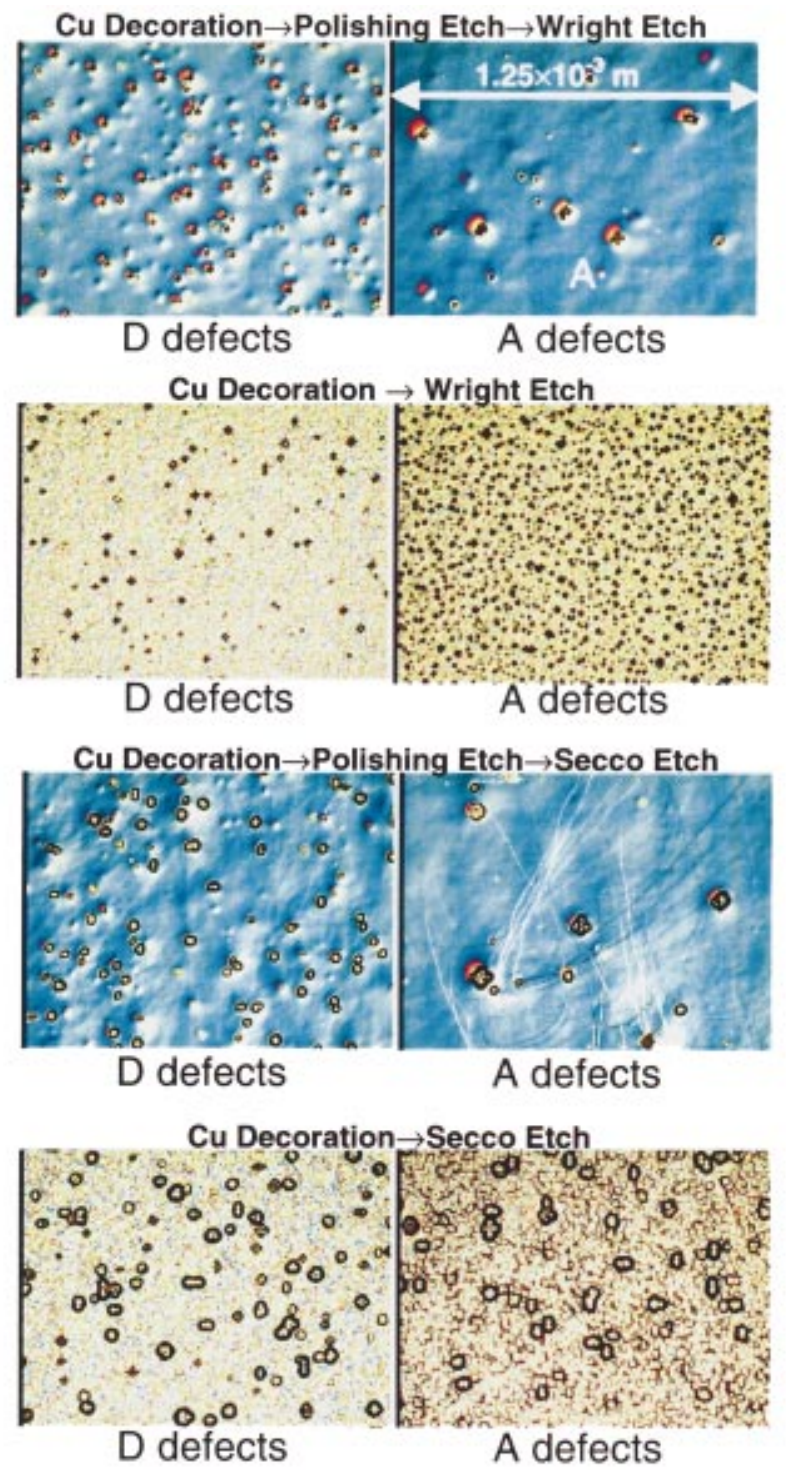

Figure 19. Effect of the surface copper precipitate colonies on the quality of microdefect decoration.

cipitates known as the surface copper precipitate colonies. Formation of the surface copper precipitate colonies is a function of the bulk microdefect density. If the bulk microdefect density is high, sufficient amount of copper precipitates on the bulk microdefects, resulting in a weak out-diffusion effect. However, when the bulk microdefect density is lower, sufficient copper out-diffuses to the wafer surface and forms high density surface colonies.

Using the principles of the proposed phenomenological model, the variation in the surface copper precipitate colony distribution as a function of the bulk-microdefect density can be revealed. For this purpose, two experiments involving different sequential processes were conducted

(i) Copper precipitation $\rightarrow$ Polishing etching $\rightarrow$ Decorating etching

(ii) Copper precipitation $\rightarrow$ Decorating etching

Consider a local section of a wafer having low density A-defects. As explained before, the standard experiment described by the first set of processes above contains a surface polishing step and, hence, allows elimination of surface disturbances caused by the surface copper precipitate colonies (Fig. 18a). However, in the second experiment, such a polishing step is absent. Since the decorating efficiency in the presence of a copper precipitate is positive for a deco- 
rating etchant, the surface copper precipitate colonies generate etch pits on the surface. Again, in the absence of a surface polishing step, these surface pits move with the moving wafer surface during further removal. When the bulk microdefect is revealed in the form of a pit by the decorating etchant, it finds itself surrounded by many pits created by the surface copper precipitate colonies (Fig. 18b).

In the D-defect region, however, the bulk-microdefect density is quite high. Hence, the effect of the surface copper precipitate colonies is insignificant. Thus, a comparison between experimental results of the first and the second experiments reveals the presence of the high density surface copper precipitate colonies only in the A-defect region. Figure 19 shows that the second experiment increases the surface density of the pits in the A-defect region, while the apparent change in the density of etch pits in the D-defect region is quite negligible. These results verify the kinetic and hydrodynamic basis for identifying the polishing and the decorating etchants.

\section{Conclusions}

Chemical etching is widely applied to decorate microdefects in monocrystalline silicon. Etchants used for defect decoration are typically known as decorating etchants. Chemical etching is also widely applied to decrease the surface roughness of silicon wafers. Generally, etchants used in this application are known as polishing etchants. The interplay between the mass-transport effects and the surface kinetics in chemical etching of monocrystalline silicon can be quantified to define decorating and polishing capacities of an etchant. The nature of an etchant, in essence, is given in terms of the time scale of the liquid-phase transport of reagents through the concentration boundary layer or the mass-transport film and that of the solid surface kinetics. The mass-transport time scale is quantified in terms of a mass-transport resistance derived from the classical filmtransport theory and the kinetic time scale is quantified by the effective kinetic resistance given by the effective surface kinetics.

The decorating etching creates pits at the microdefect sites as a result of difference between the etching rate of the microdefect site and that of the surrounding defect-free silicon. The quality of the microdefect decoration improves by precipitation of copper as $\mathrm{Cu}_{3} \mathrm{Si}$ particles around microdefects, known as a copper precipitate colonies. The decorating efficiency of an etchant at the microdefect site, defined as the ratio of rate of increase in the microdefect related etch-pit depth to the maximum possible rate of increase in the pit depth, can be given as a function of the effective kinetic and masstransport resistances at the site and on the perfect silicon surface. The polishing efficiency of an etchant, defined as the ratio of rate of decrease in the pit depth to the maximum possible rate of decrease, is also given as a function these resistances.

Although the variation of the decorating efficiency as a function of the ratio of effective mass transport resistance to the effective kinetic resistance is complex, generally, the decorating efficiency decreases with an increase in this ratio. The decorating efficiency is zero when the sum of effective kinetic and effective mass-transport resistances at the microdefect site is equal to the sum of the effective kinetic and mass-transport resistances on the perfect silicon surface. The crossover from decorating conditions to polishing conditions can occur by increasing the mass-transport effects.

The effective mass-transport resistance is a function of two parameters, the effective liquid-phase diffusivity of reagents and the mass-transport film thickness. Two limiting cases studied by fixing one parameter while varying the other reveal two different behaviors. Although the asymptotic behaviors of the fixed diffusivity and the fixed film thickness cases are quite different, generic behavior of the decoration intensity and the crossover criteria remain the same.

In the presence of a weak polishing etchant, when the masstransport effects are present but not dominant, microdefect decoration can exhibit autoinhibition. Under autoinhibiting conditions, weak microdefect decoration takes place by the formation of shallow etch pits. However, as the etch-pit depth increases, the decorating efficiency decreases. The etch pits are eventually erased after complete dissolution of the copper precipitate colonies due to the polishing nature of the etchant in the absence of the copper precipitates. Under autoinhibition, a site can make a transition from microdefect decorating conditions to neutral conditions even before complete dissolution of copper precipitates. The maximum dynamic etch-pit depth at this transition under autoinhibiting conditions is given as a function of the microdefect kinetics, the surface kinetics, and the liquid-phase diffusivity.

The propagation of etch pits is as important as their formation for an efficient microdefect decoration. Therefore, kinetic effects must be very dominant over mass-transport effects to achieve an efficient microdefect decoration.

Potentially decorating and polishing etchants can be classified based on their dependence on the reactor hydrodynamics. The etching rates of decorating etchants show a weak dependence on the hydrodynamics in the reactor whereas the etching rates of polishing etchants show a strong dependence on the reactor hydrodynamics. The surface irregularities are preserved by a decorating etchant whereas a polishing etchant erases surface irregularities.

\section{Acknowledgments}

The authors thank Shawn Patton and Steve Mecker for performing experiments. The authors also acknowledge Steve Kimbel and John Pitney for their suggestions to improve the quality of the presentation.

MEMC Electronic Materials, Inc., assisted in meeting the publication costs of this article.

\section{References}

1. W. C. Dash, J. Appl. Phys., 29, 736 (1958)

2. W. C. Dash, J. Appl. Phys., 30, 459 (1959).

3. V. V. Voronkov, J. Cryst. Growth, 59, 625 (1982).

4. F. Shimura, Semiconductor Silicon Crystal Technology, p. 56, Academic Press, San Diego (1989).

5. T. Abe, T. Samizo, and S. Maruyama, Jpn. J. Appl. Phys., 5, 458 (1966).

6. A. J. R. de Kock, J. Electrochem. Soc., 118, 1851 (1971).

7. P. M. Petroff and A. J. R. de Kock, J. Cryst. Growth, 30, 117 (1975).

8. H. Föll, U. Gösele, and B. O. Kolbesen, J. Cryst. Growth, 40, 90 (1977).

9. P. J. Roksnoer and M. M. B. van den Boom, J. Cryst. Growth, 53, 563 (1981)

10. D. G. Schimmel and M. J. Elkind, J. Electrochem. Soc., 125, 152 (1978).

11. E. Sirtl and A. Adler, Z. Metallkd., 52, 529 (1961).

12. F. Secco d'Aragona, J. Electrochem. Soc., 119, 948 (1972).

13. D. G. Schimmel, J. Electrochem. Soc., 123, 734 (1976).

14. M. W. Jenkins, J. Electrochem. Soc., 124, 757 (1977).

15. K. H. Yang, J. Electrochem. Soc., 131, 1140 (1984).

16. W. C. Dash, J. Appl. Phys., 27, 1193 (1956).

17. A. J. R. de Kock, Acta Electron., 16, 303 (1973).

18. A. J. R. de Kock, Appl. Phys. Lett., 16, 100 (1970).

19. K. Graff, Metal Impurities in Silicon-Device Fabrication, p. 98, Springer-Verlag, Berlin (2000)

20. S. P. Murarka, I. V. Verner, and R. J. Gutmann, Copper-Fundamental Mechanisms for Microelectronic Applications, John Wiley \& Sons, New York (2000).

21. L. Muléstagno and B. Falster, Private communication (1999)

22. B. Schwartz and H. Robbins, J. Electrochem. Soc., 108, 365 (1961).

23. B. Schwartz and H. Robbins, J. Electrochem. Soc., 123, 1903 (1976)

24. H. Robbins and B. Schwartz, J. Electrochem. Soc., 106, 505 (1959).

25. H. Robbins and B. Schwartz, J. Electrochem. Soc., 107, 108 (1960)

26. A. F. Bogenschütz, W. Krusemark, K. H. Locherer, and W. Mussinger, J. Electrochem. Soc., 114, 970 (1967).

27. D. L. Klein and D. J. D'Stefan, J. Electrochem. Soc., 109, 37 (1962).

28. D. R. Turner, J. Electrochem. Soc., 108, 561 (1961).

29. K. Gaffney and H. Chiou, Abstract 502, The Electrochemical Society Meeting Abstracts, Vol. 98-1, San Diego, CA, May 3-8, 1998.

30. Th. Bauer, L. Farbry, T. Teuschler, G. Schwab, and M. Stadler, Abstract 321, The Electrochemical Society Meeting Abstracts, Vol. 98-1, San Diego, CA, May 3-8, 1998.

31. J. P. John and J. McDonald, J. Electrochem. Soc., 140, 2622 (1993).

32. D. J. Monk, D. S. Soane, and R. T. Howe, J. Electrochem. Soc., 141, 264 (1994).

33. D. J. Monk, D. S. Soane, and R. T. Howe, J. Electrochem. Soc., 141, 270 (1994)

34. D. J. Monk, D. S. Soane, and R. T. Howe, Thin Solid Films, 232, 1 (1993).

35. D. J. Monk and D. S. Soane, J. Electrochem. Soc., 140, 2339 (1993).

36. M. S. Kulkarni and H. F. Erk, J. Electrochem. Soc., 147, 176 (2000).

37. O. Levenspiel, Chemical Reaction Engineering, 2nd ed., p. 349, Wiley Eastern Limited, New Delhi (1972)

38. R. H. Perry and D. W. Green, Chemical Engineer's Handbook, 6th ed., p. 4.1 McGraw-Hill, New York (1984).

39. E. L. Cussler, Diffusion-Mass Transfer in Fluid Systems, Cambridge University Press, Cambridge (1984). 